\title{
Leveraging Insights from Mobile App Reviews to Support Release Planning and Maintenance
}

\author{
By \\ Junaid Maqsood \\ A thesis submitted to \\ the Faculty of Graduate and Postdoctoral Affairs \\ in partial fulfilment of \\ the requirements for the degree of \\ Master of Computer Science \\ Ottawa-Carleton Institute for Computer Science \\ School of Computer Science \\ Carleton University \\ Ottawa, Ontario \\ January 2018 \\ (C) Copyright \\ 2018, Junaid Maqsood
}


The undersigned hereby recommend to the Faculty of Graduate and Postdoctoral Affairs acceptance of the thesis,

Leveraging Insights from Mobile App Reviews to Support Release

Planning and Maintenance

submitted by

Junaid Maqsood

Dr. Douglas Howe

(Director, School of Computer Science)

Dr. Olga Baysal

(Thesis Supervisor)

Dr. Anthony White

(Internal Examiner)

Dr. Ana-Maria Cretu

(External Examiner)

Carleton University

January 2018 


\begin{abstract}
Developers seek to know what the users of the mobile apps they developed are saying about their products and what improvements they want. This helps developers update their apps according to the users' feedback and need. Extracting such information from the reviews can be a tedious and time-consuming task if done manually. The goal of this thesis is to automate the process of leveraging key insights from the users' reviews to help developers better understand key concerns of large user population and plan future releases of their apps. In this thesis, we present an approach that automates the process of classifying user reviews into five categories according to the information contained within them. This information can range from improvements about the app to reporting bugs along with how users like/dislike the app. We then group related reviews into clusters by utilizing the Latent Dirichlet Allocation (LDA) based topic modelling and vector-space model. Then, for each generated cluster we extract the overall sentiment, generate hot topics and a short summary. We evaluate different steps of our technique on a variety of data (sampled from our original dataset of 14,000 reviews for 35 mobile apps), depending on the context. Our technique achieved the overall accuracy of $81.5 \%$ in classifying user reviews into five categories and the overall accuracy of $78.67 \%$ in generating clusters of related reviews.
\end{abstract}




\section{ACKNOWLEDGEMENTS}

I would like to express my deepest gratitude to my supervisor, Dr. Olga Baysal, whose expertise, guidance and support made it possible for me to work on such an amazing topic. I would also like to thank the School of Computer Science of Carleton University for giving me an opportunity to come and study in such an amazing environment.

In addition, I would also like to thank all my teachers who believed in me and who helped me throughout the various aspects of life. I would also express my heartfelt gratitude towards my previous supervisor Prof. Sarmad Ali, who helped and made it possible for me to come to Canada and study at Carleton University.

I would also express my gratitude towards all my teachers that made it possible for me to become who I am today and to achieve such an amazing milestone in my life. I would also like to thank all my friends and colleagues for their encouragement and moral support that made my stay in Canada more enjoyable.

Dedication : I would like to dedicate this thesis to my parents, without their love and support this would not have been possible. Their moral support is what helped me in the tough times. Especially to my Father who made it all possible and encouraged me to do what I loved and to study in such an amazing part of the world.

\section{Thank You!}




\section{Contents}

1 Introduction 2

1.1 Motivation for the Thesis . . . . . . . . . . . . . . . . 2

1.2 Objectives of the Thesis . . . . . . . . . . . . . . 3

1.2.1 $R Q_{1}$ : How accurate is our technique in classifying user reviews according to different categories? . . . . . . . . . . . . 4

1.2.2 $R Q_{2}$ : How accurate are the review clusters generated by our technique?..................... 4

1.2.3 $R Q_{3}$ : How much time, on average, does our technique save when compared to a manual process of review-based development planning? . . . . . . . . . . . . . . . . . 4

1.2.4 $R Q_{4}$ : What are the hot topics that users talk about? . . . . . 4

1.3 Contributions of the Thesis . . . . . . . . . . . . 5

1.4 Organization of the Thesis . . . . . . . . . . . . . 5

2 Background and Related Work $\quad 6$

2.1 Background ...................... 6

2.2 Mining Mobile App Data . . . . . . . . . . . . . . . . 8

2.3 Sentiment Analysis . . . . . . . . . . . . . . . . . . . . . 9

2.4 Latent Dirichlet Allocation . . . . . . . . . . . . . . . . . . . 10

2.5 Finding Similarities . . . . . . . . . . . . . . . . . . . . 10

3 Methodology 12

3.1 Data Collection and Dataset . . . . . . . . . . . . . 13 
3.2 The Main Algorithm of Our Technique . . . . . . . . . . . . . 16

3.2.1 Implementation Details . . . . . . . . . . . . . . . . 17

3.3 Categorizing User Reviews . . . . . . . . . . . . . . . . . 18

3.4 Topic Modelling . . . . . . . . . . . . . . . . . . . . . 21

3.5 Sentiment Analysis . . . . . . . . . . . . . . . . . . . 22

3.6 Clustering Reviews . . . . . . . . . . . . . . . . . . . 24

3.7 Extracting Cluster Summaries . . . . . . . . . . . . . 26

3.8 Output of the Technique . . . . . . . . . . . . . . 27

3.9 Data Analysis . . . . . . . . . . . . . . . . . . 28

4 Results 30

4.1 $R Q_{1}$ : How accurate is our technique in classifying user reviews according to different categories? . . . . . . . . . . . . . . . . 30

$4.2 R Q_{2}$ : How accurate are the review clusters generated by our technique? 33

$4.3 R Q_{3}$ : How much time, on average, does our technique save when compared to a manual process of review-based development planning? . . 35

$4.4 R Q_{4}$ : What are the hot topics that users talk about? . . . . . . . 36

5 Discussion $\quad 40$

5.1 Comparative Analysis . . . . . . . . . . . . . . . . . 40

5.2 Implications for Developers . . . . . . . . . . . . . . 41

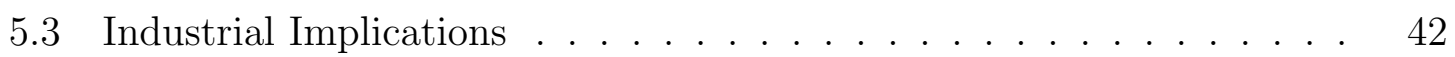

5.4 Implications for Future Research . . . . . . . . . . . . . . 43

5.5 Threats to Validity . . . . . . . . . . . . . . . . . 44

6 Conclusions $\quad 46$

6.1 Summary of the Results . . . . . . . . . . . . . . 46

6.2 Future Work . . . . . . . . . . . . . . . . . 47

$\begin{array}{ll}\text { A Tables } & 48\end{array}$

B Figures $\quad 55$

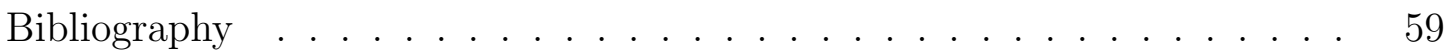




\section{List of Tables}

3.1 Top categories based on the cumulative number of apps. . . . . . . . 14

3.2 Top categories based on the distinct number of apps. . . . . . . . . 15

3.3 Categorizing user reviews. . . . . . . . . . . . . . . 19

3.4 Sentiment categories. . . . . . . . . . . . . . . 22

3.5 Datasets used in research questions. . . . . . . . . . . . 28

4.1 Examples of classifying user reviews. . . . . . . . . . . . . . . 31

4.2 Classification accuracy of user reviews. . . . . . . . . . . . 33

4.3 Accuracy for cluster generation (single app study) . . . . . . . . . 35

4.4 Hot topics and summarization. . . . . . . . . . . . . 39

A.1 Sample app reviews. . . . . . . . . . . . . . . . . . . . 48

A.2 Selected 35 apps details. . . . . . . . . . . . . . . 54 


\section{List of Figures}

3.1 Overview of our technique. . . . . . . . . . . . . . . . 13

4.1 Distribution of reviews vs. distribution of clusters per category. . . . 32

4.2 Distribution of clusters. . . . . . . . . . . . . . . . . . . 34

4.3 Sample cluster results. . . . . . . . . . . . . . . . . . 38

B.1 Cluster results, Page $1 \ldots \ldots \ldots \ldots 5$

B.2 Cluster results, Page $2 \ldots \ldots \ldots 6$

B.3 Cluster results, Page $3 \ldots \ldots \ldots \ldots$

B.4 Cluster results, Page $4 \ldots \ldots \ldots \ldots$ 


\section{ACRONYMS}

$\begin{array}{ll}\text { TF-IDF } & \text { Term Frequency-Inverse Document Frequency } \\ \text { VSM } & \text { Vector Space Model } \\ \text { UX } & \text { User Experience } \\ \text { OTS } & \text { Open Text Summarizer } \\ \text { RQs } & \text { Research Questions } \\ \text { LDA } & \text { Latent Dirichlet Allocation } \\ \text { RSS } & \text { Really Simple Syndication } \\ \text { CLAP } & \text { Crowd Listener for releAse Planning } \\ \text { VS } & \text { Vector Space } \\ \text { PH } & \text { Person Hours }\end{array}$




\section{Chapter 1}

\section{Introduction}

In this chapter, we first state our motivation for this thesis (Section 1.1). Section 1.2 outlines our key objectives in a form of four research questions. Section 1.3 offers a list of contributions of this thesis. And finally, Section 1.4 presents the overall structure of the thesis.

\subsection{Motivation for the Thesis}

App support is an essential part of improving and debugging apps that exist in different app stores. App users write and submit reviews providing their feedback on the app. This feedback can include requests for new features or reports of various bugs that need to be fixed. While such user feedback is valuable to the development teams, the sheer volume of daily reviews can be overwhelming, e.g., everyday hundreds of reviews are posted by different users for an app [1]. User reviews are essential for extracting insights into what the users want in the future and how an app can be improved. This information can also be helpful in attracting more users, as well as making them feel appreciated. However, the challenge here is that in order for the developers to make sense of these reviews and understand what users want, they must read each review one by one making this a time-consuming task. To help developers overcome this challenge and assist them in "making sense" of user reviews, we develop a technique that extracts and aggregates useful information from reviews without the 
need of developers reading every single review.

\subsection{Objectives of the Thesis}

In this thesis, we propose a technique that can help developers in extracting the information they need from the reviews of their apps. Our approach aims at categorizing the reviews by classifying and clustering them using state of the art techniques. It allows developers to determine the "hot topics" that users are discussing without the need of reading all the reviews. We collected 14,000 reviews for the top 35 randomly selected apps. This dataset will be used to evaluate our technique.

We now provide a brief overview of our approach. First, our technique categorizes user reviews according to their type such as bug report, feature request, user experience, rating or other. We then perform a sentiment analysis on the reviews to extract user sentiment. After that, we assign each review a topic by applying Latent Dirichlet Allocation (LDA), a topic modelling technique that extracts topics from a given text [2].

We found that the review title provided by the user contains useful information about what the user is trying to convey. We combine the assigned topic with the title of the review and represent it as a vector using the Term Frequency-Inverse Document Frequency (TF-IDF) algorithm [3]. The vectors within the Vector Space Model (VSM) [4] are grouped together based on cosine similarity. Similar vectors here represent related reviews, therefore, we group all related reviews together in a cluster and create a heading based on the topics. We also assign each cluster with an overall sentiment label based on the majority vote, i.e., what the majority sentiment of the reviews in that cluster is. We then sort the clusters based on the number of reviews within them to generate hot topics. Finally, we apply the Open Text Summarizer (OTS) algorithm [5] that generates a short summary for each cluster. This summary consists of the important sentences within the reviews in a cluster.

In this thesis, we aim at answering the following Research Questions (RQs) : 


\subsection{1 $R Q_{1}$ : How accurate is our technique in classifying user reviews according to different categories?}

We assess the accuracy of our classification technique in assigning reviews to a particular category such as bug report, feature request, user experience, rating or other. We compare the results of our automated categorization technique with the results of our manual categorization of the reviews.

\subsection{2 $R Q_{2}$ : How accurate are the review clusters generated by our technique?}

We evaluate the accuracy of the generated clusters by manually inspecting them and checking the number of correctly placed reviews within each cluster.

\subsection{3 $R Q_{3}$ : How much time, on average, does our technique save when compared to a manual process of review- based development planning?}

We calculate the time it takes for us to perform the manual classification of the reviews in $R Q_{1}$ and the manual inspection of the clusters in $R Q_{2}$. We then compare this time with the time it takes for our technique to generate the results for $R Q_{1}$ and $R Q_{2}$ for the same datasets.

\subsection{4 $R Q_{4}$ : What are the hot topics that users talk about?}

For the planning of next releases, we identify various hot topics. These topics define the major themes that the users are talking about within their reviews. By determining hot topics and extracting their summaries, our technique recommends developers the key areas for improving their apps and maximizing the user experience. 


\subsection{Contributions of the Thesis}

This thesis makes the following contributions:

- We provide a technique that automatically categorizes user reviews and clusters related reviews according to their topics. Our technique is able to determine not only hot topics but also provide a summary of hot topics to support developers with a concise information about user requests.

- Through an empirical study we evaluate various steps of our technique assessing its various aspects (e.g., accuracy in classifying user reviews, accuracy of generated clusters, etc.).

- We made our dataset publicly available (will be provided at https://www. junaidmaqsood. com/LI-MAR-S-RPAM).

\subsection{Organization of the Thesis}

The rest of the thesis is organized as follows. Chapter 2 provides an overview of the related work along with introduction to the various concepts used in this study. Chapter 3 discusses the methodology we followed in our study including a detailed summary of how we collected our data and what methods we used on the data that lead to our results. Chapter 4 reports the results of our study. In Chapter 5 we discuss in detail our results in comparison to previous studies and also talk about various implications for developers, industry practitioners, as well as researchers. Section 5.5 discusses threats to validity. And finally, Section 6 concludes the thesis and outlines possible future directions. 


\section{Chapter 2}

\section{Background and Related Work}

In this chapter, we provide a background on the mobile platforms (Section 2.1), discuss relevant and related research in the area of mining mobile app data (Section 2.2), as well as explain the tools we employed in our work (Sections 2.3, 2.4, and 2.5).

\subsection{Background}

In the recent years, there has been a considerable progress in mobile technology. Nowadays, nearly every company provides services or products to their customers via mobile apps. However, when it comes to the app distribution, there are only a few giants in the market such as Apple's App Store [6], Google's Play Store [7] and the Amazon Appstore [8]. These platforms provide a wide range of services to developers for managing and distributing their apps. Also, these platforms provide developers with tools to engage in user experiences. Users can provide ratings or reviews as a feedback on their experience with an app.

When it comes to the app quality, developers are striving to increase user population and engagement by finding and fixing bugs or by offering effective improvements to their apps. When it comes to the reviews, users tend to write useful information that can tell the developer how to improve the app [9]. User reviews have been stated to be the "voice of the users" in many previous research works $[10,11,12]$. However, some reviews are composed of spam, paid advertisements and are not so useful 
information [13].

Leveraging user feedback from the reviews is, therefore, an interesting and important problem. Reading all the reviews and figuring out what users are trying to say is one way of tackling the problem. However, for most of the successful apps this approach is not feasible due to the sheer volume of reviews that are being posted every day. Many apps receive tens and even hundreds of reviews on a daily basis. According to Pagano et al. [1], users submit, on average, 22 reviews per day per app. Therefore, a better way is required to organize, manage and extract insights from the reviews.

To tackle the issue of high volume of the reviews being published every day, there is a need to organize these reviews in a useful and manageable way. There have been multiple studies in the past $[14,15,16,17]$ that focused on the value-related features. Davod et al. [17] defined these value-related features as certain features of the software that may encourage or discourage users to prefer other features (i.e., a value of a particular feature might be positively or negatively influenced by other features). They proposed an approach that studied such features and tried to find the optimal subset of the features based on user feedback. This is important to better plan next release. As some features being provided, the automatic release planning might also require some other dependent functionalities.

Villarroel et al. [18] have recently proposed a tool that utilizes the export reviews feature of the Google's Play to help developers plan the release change log. Their tool uses multiple machine learning approaches to determine what people are talking about. They started with training a classifier from manually classified user reviews. This classifier was then able to classify with an accuracy of $86 \%$ the reviews into one of the three categories (i.e., bug reporting, feature suggestion, other). Afterwords, they clustered similar reviews within one classification using a Vector Space model. From these clusters they provided a list of most important requests or reports by the user.

In this work, we propose a similar technique with certain improvements (extended classification of review categories, sentiment analysis, and summarization of topics) and try to utilize various state-of-the-art techniques (e.g., topic modelling [2]) to 
leverage insights from user reviews. Unlike the previous study [18], our dataset consists of the reviews collected from a different app store (i.e., Apple's App Store). Our technique is also able to utilize automated summarization techniques to skim the reviews into a summary. Along with the summary, our clusters also contain sentiment for both the cluster, as well as the reviews within it. We also utilize this sentiment to better analyze the results and to better plan next releases.

\subsection{Mining Mobile App Data}

Several approaches have aimed to use the information provided by the reviews to find useful information. Charrada [19] studied the factors that influence the usefulness of reviews and found 32 factors that determine how useful a review is; these factors are arranged into five categories (i.e., language, volume and longevity, rating and sentiment, content, and reviewer). Chen et al. [20] developed a tool called $A R$ miner to classify reviews as either informative or not. Many studies [10, 21, 22] have used various machine learning techniques to support release planning. Panichella et al. [9] used a combination of natural language processing with sentiment analysis to classify reviews into five generic categories (i.e., information giving, information seeking, feature request, problem discovery, and others).

User app reviews have also been linked with various other development issues. Ciurumelea et al.'s study [23] focused more on specific hardware-related issues from the user reviews. They presented a novel approach which organized user reviews into various hardware issues (e.g., performance, device, battery, security and so on). Studies have also tried to link app reviews with various other artifacts. Johann et al. [24] found features from the app description and linked those to the reviews. Nayebi et al. [25] linked release planning with information on social media (e.g., Twitter) and used that to find issues and feature requests. Mercado et al. [26] combined user reviews from different platforms (i.e., Google and Apple Store) for the same app to find issues related to cross-platform development.

Maalej et al.[27] presented a keyword based approach that used specific humandefined features to classify a specific reviews in one of their three review category (i.e., 
Bug Reports, Feature Request, and Others). Lacob et al. [28] in 2013 presented an algorithm that utilizes linguistic rules to categorize reviews into bug reports or feature requests. They extended their study in 2016 [29] by creating a tool that utilized not only the reviews but other software artifacts like dev logs and GitHub issues and presented them to the developer in a specially designed IDE. They combined the linguistic approach of categorizing reviews with the keyword based approach. They designed their tool to perform three major tasks (i.e., automatically extract user reviews from app store, support developer's queries on the app reviews, and support management through integration with other software development tools available like GitHub[30], JIRA[31] or Bugzilla[32]).

\subsection{Sentiment Analysis}

Definition: Sentiment analysis, also called opinion mining, is the field of study that analyzes people's opinions, sentiments, evaluations, appraisals, attitudes, and emotions towards entities such as products, services, organizations, individuals, issues, events, topics, and their attributes [33].

Sentiment analysis is widely applied to voice of the customer materials such as reviews and survey responses, online and social media, and healthcare materials for applications that range from marketing to customer service to clinical medicine. Sentiment analysis has been effectively applied to mine user opinions (i.e., sentiment). However, most of the previous models for sentiment analysis are build on Twitter data $[34,35,36,37]$. Using these models in our study could lead to a lower accuracy and many wrongly classified instances [38]. Creating a more specific model could have solved this problem; however, this requires manually labelled data for app store reviews. We decided to employ a word-based approach instead and used the SentiStrength Java library [39] to mine the sentiment. Such an approach provides two scores based on the words used within the text - one for positive ones and the other for negative ones. 


\subsection{Latent Dirichlet Allocation}

Definition: In natural language processing, Latent Dirichlet Allocation (LDA) is a generative statistical model that allows sets of observations to be explained by unobserved groups that explain why some parts of the data are similar. For example, if observations are words collected into documents, it posits that each document is a mixture of a small number of topics and that each word's creation is attributable to one of the document's topics. LDA is an example of a topic model and was first presented as a graphical model for topic discovery by David Blei, Andrew Ng, and Michael I. Jordan in 2003 [2].

In order to extract topics from the reviews we use the LDA model [2]. Many previous studies have adopted LDA to find very useful information out of the reviews. Some research focused on finding out negative topics and checking why people hate the app [40], how the new update is liked by the users [10] or analyzing feature requests [41] and app behaviour based on the topics extracted by the LDA technique [42].

\subsection{Finding Similarities}

To compute similarities among different reviews, we employ a technique used in the past for various recommender systems [43]. We use $T F-I D F$ on the review's topics and the review titles. Then, based on these document vectors we can use cosine similarity to group similar ones together. This technique has worked well to find relevant topics for the user combined with the user behaviour.

$T F-I D F$. In information retrieval, tfidf or TFIDF, short for term frequencyinverse document frequency, is a numerical statistic that is intended to reflect how important a word is to a document in a collection or corpus [44]. It is often used as a weighting factor in searches of information retrieval, text mining, and user modeling. The tf-idf value increases proportionally to the number of times a word appears in the document, but is often offset by the frequency of the word in the corpus, which helps to adjust for the fact that some words appear more frequently in general. Nowadays, tf-idf 
is one of the most popular term-weighting schemes; $83 \%$ of text-based recommender systems in the domain of digital libraries use tf-idf [45]. 


\section{Chapter 3}

\section{Methodology}

To address our research questions, we followed the process shown in Figure 3.1 that consists of the following phases: 1) data collection and 2) leveraging insights. In the Phase 1, our technique is more focused on organization and collection of the data. We start with gathering all information regarding the apps and their reviews from our dataset. Then, in Phase 2, these collected reviews are passed through different modules to a) categorize them into various categories, b) employ LDA to find the most important words, i.e., topics from these reviews, and finally c) perform sentiment analysis on them. After having a well organized and completely labelled review dataset, we apply a Vector Space Model for calculating the TF-IDF Vector and the cosine similarity among them. Lastly, based on this cosine similarity we group similar reviews together into clusters.

This chapter is further organized according to the various modules that our technique consists of. We start with describing how we gathered and filtered our dataset (Section 3.1). Section 3.2 presents the overall view of our main algorithm. We define how various modules are connected to each other. Then, in Sections 3.3, 3.4, 3.5, 3.6, and 3.7, we offer the details for each module of the main algorithm. In Section 3.8, we discuss the output of our technique. And finally, Section 3.9 defines how we used different parts of our dataset to answer the research questions. 


\subsection{Data Collection and Dataset}

Apple provides an Really Simple Syndication (RSS) feed ${ }^{1}$ to search for data related to its App store. This includes searching for the different apps within different categories all around the world. We used this RSS feed to gather the data about apps and their reviews. Since each app may have hundreds and even thousands of user reviews, mining all of them is not feasible.

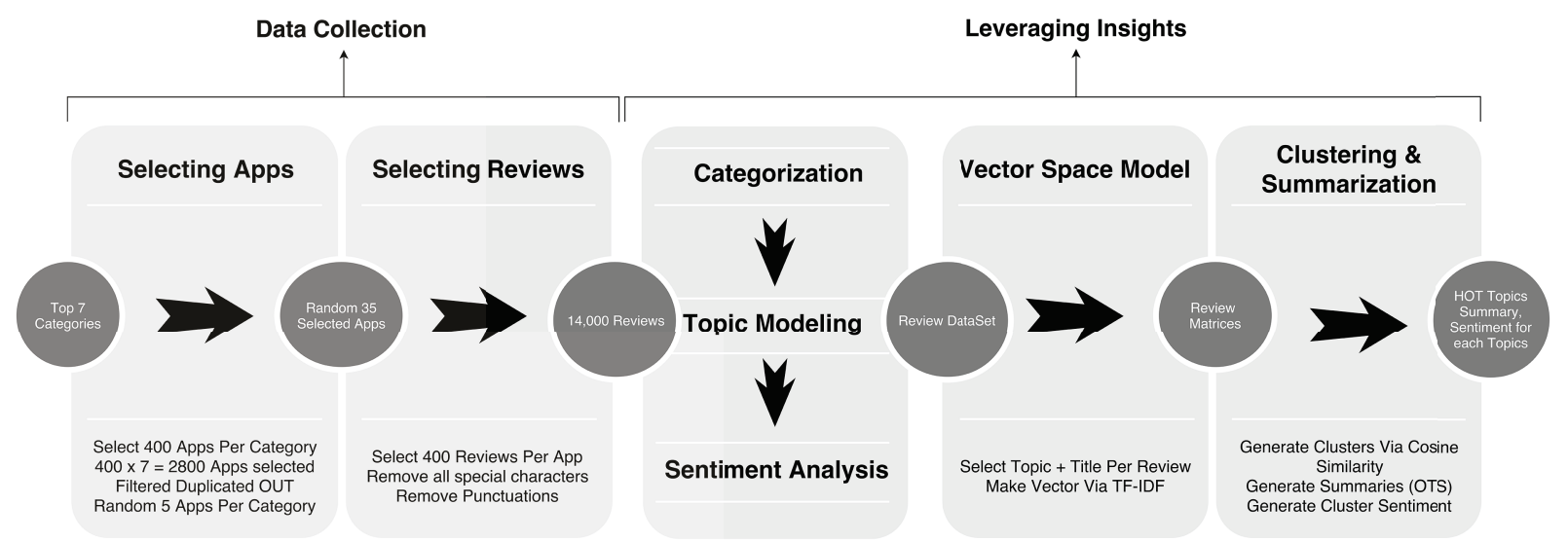

Figure 3.1: Overview of our technique.

Therefore, we decided to use a sampling formula for an unknown population size for determining our sample size of user reviews:

$$
n=\frac{Z_{a / 2}^{2} * p(1-p)}{E^{2}},
$$

where $n$ is sample size, $Z_{a / 2}$ is the $\mathrm{z}$ score for the confidence level and $p$ is the maximum variability of the population, and $E$ is the sampling error rate.

For this study, we considered a confidence level of $95 \%$, therefore $Z_{a / 2}$ becomes 1.96; $p$ is the maximum variability of the population, i.e., at $50 \%$, therefore, $p=0.5$ and finally the sampling error rate $(E)$ is of $5 \%$. The sample size $(n)$ was calculated to be 384.16 , we rounded this to 400 to simplify the process of review collection. In order to collect this sample data, we utilized the Apple's RSS feed. However, Apple

\footnotetext{
${ }^{1}$ https://rss.itunes.apple.com/en-us; accessed on July 15, 2017
} 
places multiple restrictions on their RSS feed such as: 1) a limit on the history of the data you are requesting; 2) a limit of 300 items per query.

Table 3.1: Top categories based on the cumulative number of apps.

\begin{tabular}{ccc}
\hline \multicolumn{3}{c}{ World Data (155 countries) } \\
\hline Rating & Category & \# Apps \\
\hline 1 & Games & 16,516 \\
2 & Social Networking & 2,838 \\
3 & Health \& Fitness & 1,298 \\
4 & Productivity & 1,246 \\
5 & Photo \& Video & 1,246 \\
6 & Education & 1,086 \\
7 & Music & 1,048 \\
8 & Entertainment & 948 \\
9 & Utilities & 763 \\
10 & Business & 653 \\
\hline
\end{tabular}

Category selection. Apple's RSS feed provides a wide range of categories for the app. For the purpose of this study, we decided to restrict this data to a few specific categories. App has a different store for each country. There are 155 registered countries store provided by Apple. Each store has its own top rated apps and its own reviews and user base. Majority of the apps belong to multiple stores (e.g., a game might be available in all 155 stores). For the selection of the categories we started with fetching the 400 top apps (200 for free and 200 for paid apps) across the world (i.e., 155 countries). We made two different tables, where Table 3.1 shows the top categories when we add the number of app cumulatively (i.e., if an app is present in the United States (US) store, as well as Canada (CA) store it counts as two apps), while the Table 3.2 shows the top categories when an app is only counted once no matter how many counties it belongs to. We see that the rating is different based on how we count the number of apps. Since we are more interested in finding the top categories and not how many different apps there are within the categories, we went in selecting the top 7 categories from the Table 3.1. We considered the top categories because we focused on the apps with high volume of reviews being posted daily. 
App selection. Based on the Table 3.1, we selected top 7 app categories for out initial data as this seems to be promising based on the number of apps within each category. After that, we selected United States and Canada as our primary countries (please note that our technique works globally too). We fetched the data for top 400 apps for these two counties for the seven top categories. Finally, we randomly selected 5 apps from each app category from the fetched 2,800 apps. In the end, we were left with a total of 35 apps. Table A.2 reports the list of final selected apps. The table is divided among the 7 categories and within each category we have 5 apps. For each app in the table we provide the Name, Price and the Release Date. In our actual dataset we had all the meta-data provided by the Apple's RSS feed. However, for this study we only displayed the core properties.

Table 3.2: Top categories based on the distinct number of apps.

\begin{tabular}{ccc}
\hline \multicolumn{3}{c}{ World Data (155 countries) } \\
\hline Rating & Category & \# Apps \\
\hline 1 & Games & 2,832 \\
2 & Social Networking & 425 \\
3 & Education & 384 \\
4 & Utilities & 366 \\
5 & Photo \& Video & 347 \\
6 & Entertainment & 296 \\
7 & Productivity & 278 \\
8 & Health \& Fitness & 258 \\
9 & Music & 214 \\
10 & Lifestyle & 214 \\
\hline
\end{tabular}

Reviews selection. The RSS feed provides review data in pages, each page contains 50 most recent reviews. As per the sampling size formula, we need to gather 400 reviews per each app. We fetched 8 pages of reviews for each app, and as a result we collected the total of 14,000 reviews for 35 apps forming our original dataset. In order to maintain some quality within the reviews we removed all non-English reviews or any reviews that were not written via the traditional QWERTY keyboard. For each of these reviews, we considered all the meta information including but not 
limited to the app's details, author of review, review category (i.e, computed by our technique), content (i.e, the actual review written by the user/author), rating, title, and sentiment (i.e, calculated by our technique). We have added the Table A.1 to Appendix that shows a sample of these 14,000 reviews. We could not include all the reviews due to space constraints. For this table we selected one random review per review category for each app.

\subsection{The Main Algorithm of Our Technique}

Now we discuss the main algorithm that implements our technique. It is divided into small problems. Globally, the algorithm calls different small problem solvers. Each of the problem solver (i.e., a function) is discussed in detail in further sub-sections. The overview of our technique is presented in Algorithm 3.2 that describes how we organized and implemented our approach of mining user reviews. The output of this algorithm are the generated clusters. Each cluster consists of all the reviews, title (heading of the cluster), summary of the cluster, category/type of the cluster and the sentiment of the cluster. These clusters allow developers to gain insights into the key issues users discuss in their reviews. For example, one cluster can be pointing out an issue regarding a login feature or some other new feature wanted by multiple users. However, having multiple clusters does not help us with answering the following question - which cluster is more important? To determine the importance of the cluster (here we call such clusters as "hot topics"), we take into account the number of reviews within the cluster along with its overall sentiment. If the cluster is of a negative sentiment and belongs to the bug request category, such cluster should be promptly addressed by the developers. These important clusters or hot topics highlight the functionality that should be fixed/implemented in the next release.

We start with providing Algorithm 1 with the fetched App details and the Reviews. The algorithm goes through each review (line 2-6) and assigns them to Review Category (line 3), Topic (line 4) and Sentiment (line 5). Then, it creates an empty Cluster map (line 7) that iterates through each review and finds all reviews similar to it (line 9). Once we have matches we do not want one review to be a part of multiple 


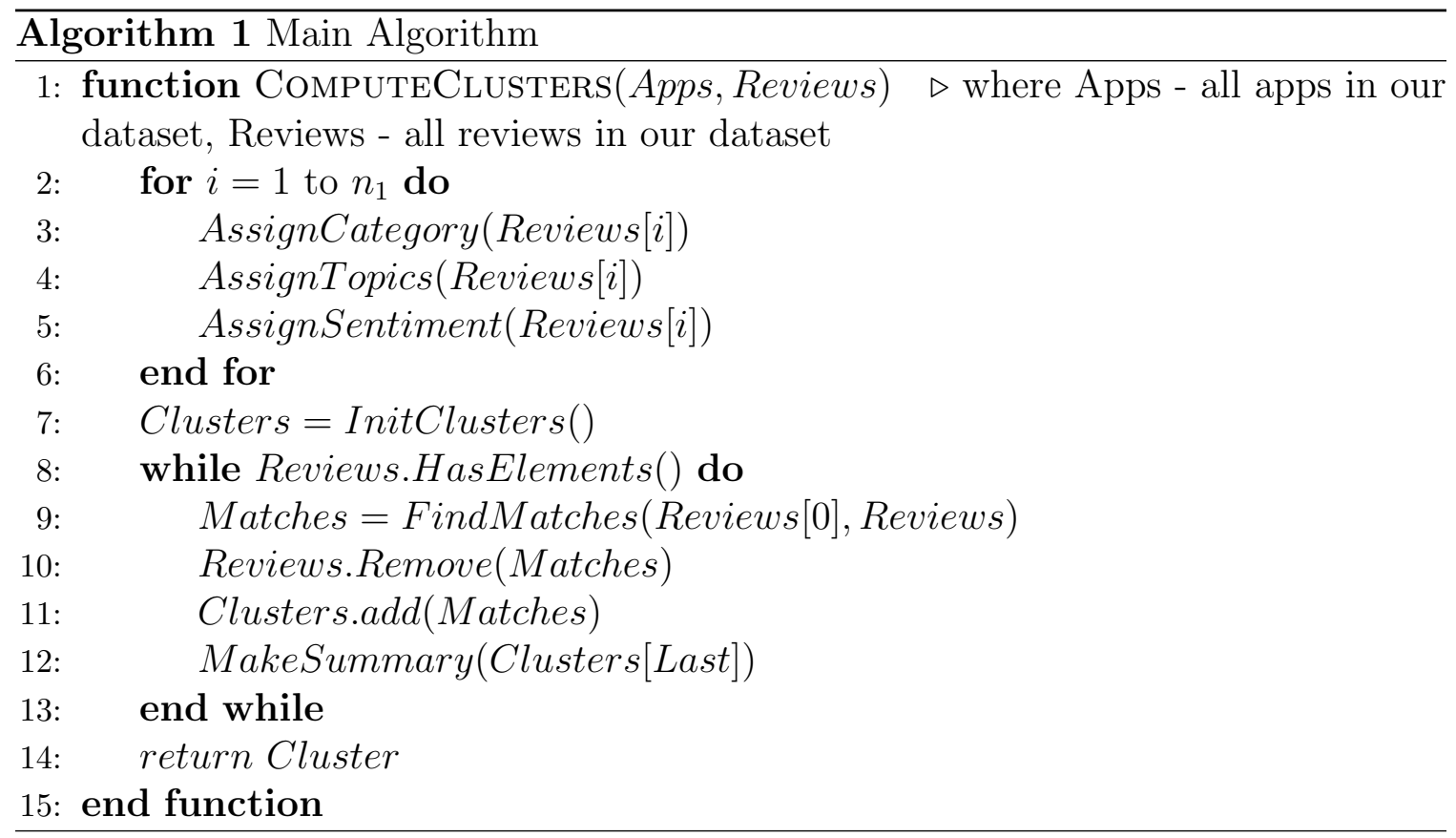

clusters. Therefore, we remove all matched reviews from the original collection (line 10). Finally we create a new cluster from the matched review and generate a summary for it (lines 11-12). Each step (function) such as AssignCategory, AssignTopics, AssignSentiment, FindMatches and MakeSummary is explained in its own subsection (see Section 3.3, Section 3.4, Section 3.5, Section 3.6, and Section 3.7, respectively).

\subsubsection{Implementation Details}

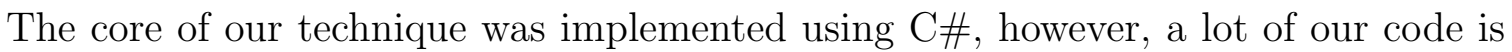
in Python. The Python script has two main functions. 1) Topic modelling that takes the reviews as input and returns a modelled topic for each review using LDA [2]. We used the "gensim" library for implementing the LDA model. 2) Sentiment analysis also takes the reviews as input and returns the sentiment strength value for each review - in between -4 and +4 . We used the SentiStrength [39] Java library for implementing the sentiment analysis of user reviews. We used PyInstaller to make the script executable. Finally, we wrote our own library for generating TF-IDF vectors and for clustering them based on the cosine similarity between vectors. All our 
modules are called from $\mathrm{C} \#$ no matter if their back end is in Python or Java.

\subsection{Categorizing User Reviews}

The first step of our process of leveraging insights from user reviews is classifying reviews into five categories: bug report, feature request, user experience, rating and other. Unlike Crowd Listener for releAse Planning (CLAP) [18], we make a more specific classification of user reviews based on our manual inspection of 1,000 reviews. We found that besides bug reports and requests for new features (two prime categories considered by Villarroel et al. [18]), users often write about their overall experience with the app, whether they liked it or not, as well as their ratings of the app. Therefore, we refined and extended the previously adopted classification of user reviews.

Keyword Extraction Based on the previous research [27, 1], for each of the category we extracted a list of keywords as shown in Table 3.3. As the previous studies were more focused on the two major review categories (i.e., Bug Reports, Feature Requests) we had to add more to the list of keywords in order for it to adopt for our class structure. We started off with traditional machine learning approaches to generate a list of features (i.e., Naive Bayes, Neural Network, SVM, Decision Trees and Random Forrest), then from these we selected the most rich features after pruning and stemming.

We applied the Porter stemming [46] to reduce all words to their root. After this, we match the reviews to the keywords according to the simple word matching algorithm [47]. If a review contains any of the keywords from Table 3.3, it is placed into the relevant category. In the situations when a review can potentially contain keywords matching multiple categories, we introduce the term priority for the categories. This priority is calculated based on how important the category is for future release planning. If a review matches both bug report, as well as user experience, it would be classified as bug report because of its higher relevance to the developers' release planning and maintenance tasks. The categories in Table 3.3 are reported according to their priority (i.e., bug report being most important one, while other being least 
Table 3.3: Categorizing user reviews.

\begin{tabular}{cl}
\hline Category & Keyword List \\
\hline Bug report & $\begin{array}{l}\text { bug, fix, freeze, fail, glitch, problem, issue, defect, crash, } \\
\text { solve, error }\end{array}$ \\
Feature request & $\begin{array}{l}\text { add, please, lack, wish, could, would, should, hope, im- } \\
\text { prove, miss, need }\end{array}$ \\
User experience & help, support, assist, when, situation, hard, easy \\
Rating & great, good, nice, very, cool, nyc, love, hate, bad, worst, \\
& worse, crap, pathetic, pathetic, fun, amazing, like, cute, \\
& super, superb, glad, best \\
Other & Any review not from above categories \\
\hline
\end{tabular}

important one).

Algorithm 2 describes the complete process of getting a review and assigning one of the five review categories from the Table 3.3 to it. It achieves that by performing the following steps:

1. Performs Pre-processing to the Review by removing all punctuation marks, tokenizing it into words and stemming each word to its basic form (line 2-6).

2. It loads all pre-defined keywords for each review category (line 7). These are the keywords presented in Table 3.3. These keywords when fetched are also stemmed using the same algorithm.

3. The algorithm also checks if the stemmed words of the reviews contain any one of the BugReport keywords from the Table 3.3 (line 8). If it does, then that review is assigned the BugReport category (line 9).

4. Similarly, if the review is not a bug report, the algorithm checks for each category keyword individually and assigns that category to it (lines 10-15).

5. If no category keyword is found, the review is assigned the Other category (lines 16-17). 


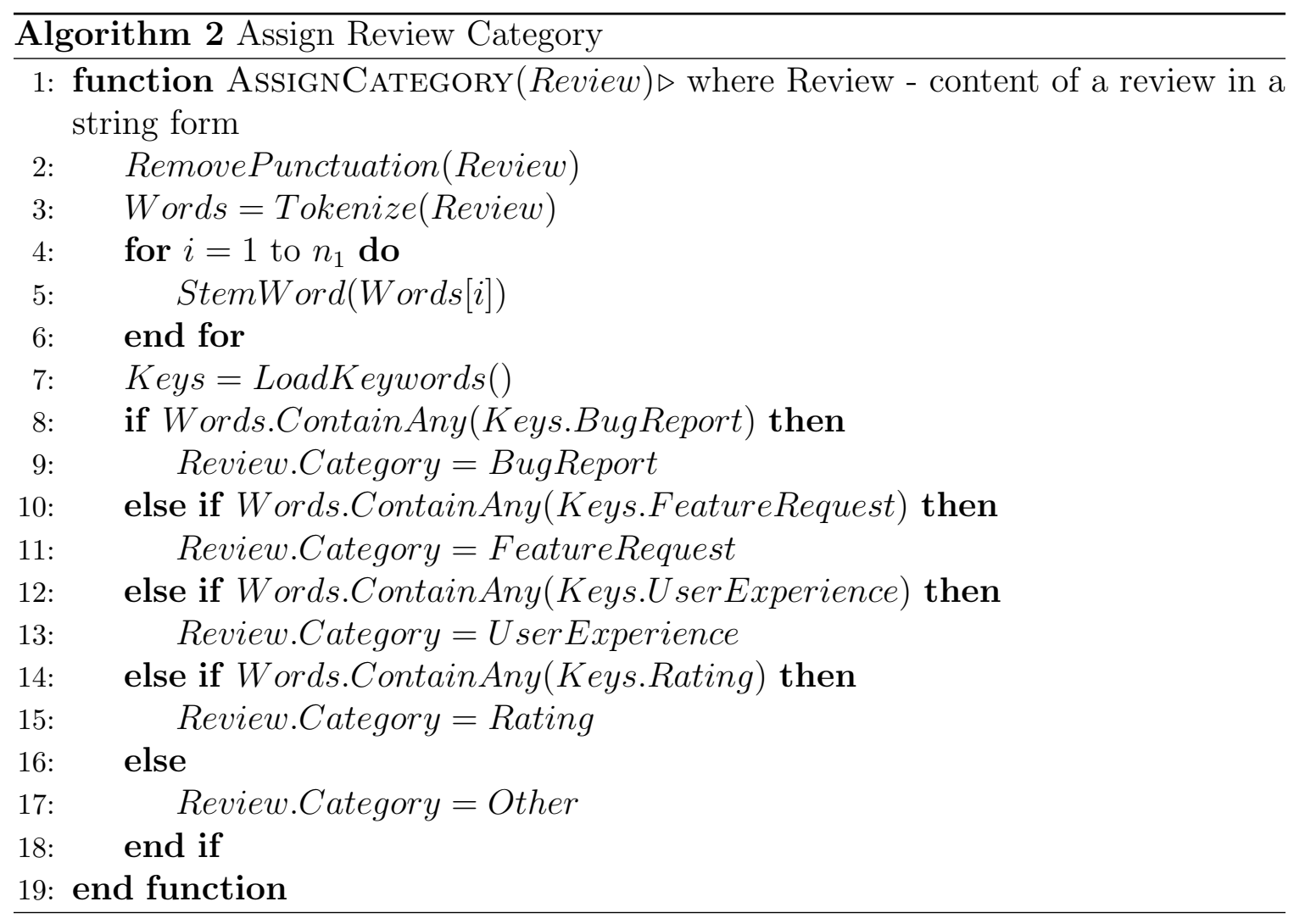




\subsection{Topic Modelling}

Topic modelling [2] is a statistical model used to capture the abstract topic for a set of documents or a text. This is a text-mining technique to discover the abstract "topics" from a collection of documents. In our study, we apply topic modelling to extract topics from user reviews. We used a LDA [2] model. LDA is a generative probabilistic model for topic modelling. The idea behind it is that every document or a text is a mixture of small topics [2]. To build a model, we start by tokenizing the text (note: stemmed words) from the user reviews, i.e., breaking them into tokens. We then apply the Python library for English stopwords list ${ }^{2}$ to remove noise from the reviews. Next, we change the tokens to $i d \leftrightarrow$ term dictionary. Then we convert tokenized documents into a document-term matrix [3]. After that, we create the LDA model that generates the topics for each review. Doing so, we noticed that the end result of this algorithm contained some generic terms as topics (e.g., app, game, ios, etc.), such words do not contribute much to the algorithm for creating clusters. To increase its efficiency we created a custom stop words list that contained words that were too generic to be considered as topics yet were not stop words. This helped the algorithm find the right words for topics.

Algorithm 3 illustrates the overall process of the topic modelling step. This part of the system is implemented in Python. We use the "nltk" and "gensim" libraries to get the topics for the reviews. The algorithm takes the review as input, removes all punctuation marks, stop words and tokenizes it (lines 2-4). Then from these tokens it stems the words based on the Porter stemming to their most basic form and converts each word to lower case (lines 5-8). The final token list is converted into a document term matrix via the nltk library and corpus (lines 9-11). The final result is provided to the gensim LDA model to create a model and generate topics for that reviews (lines 12-14). Topics represent the most important words within those reviews. We tried to use a generic model instead of doing this and trained the gensim LDA model with the publicly available dataset of Wikipedia. This resulted in creating very generic topics for the reviews.

\footnotetext{
${ }^{2}$ https://pypi.python.org/pypi/stop-words
} 


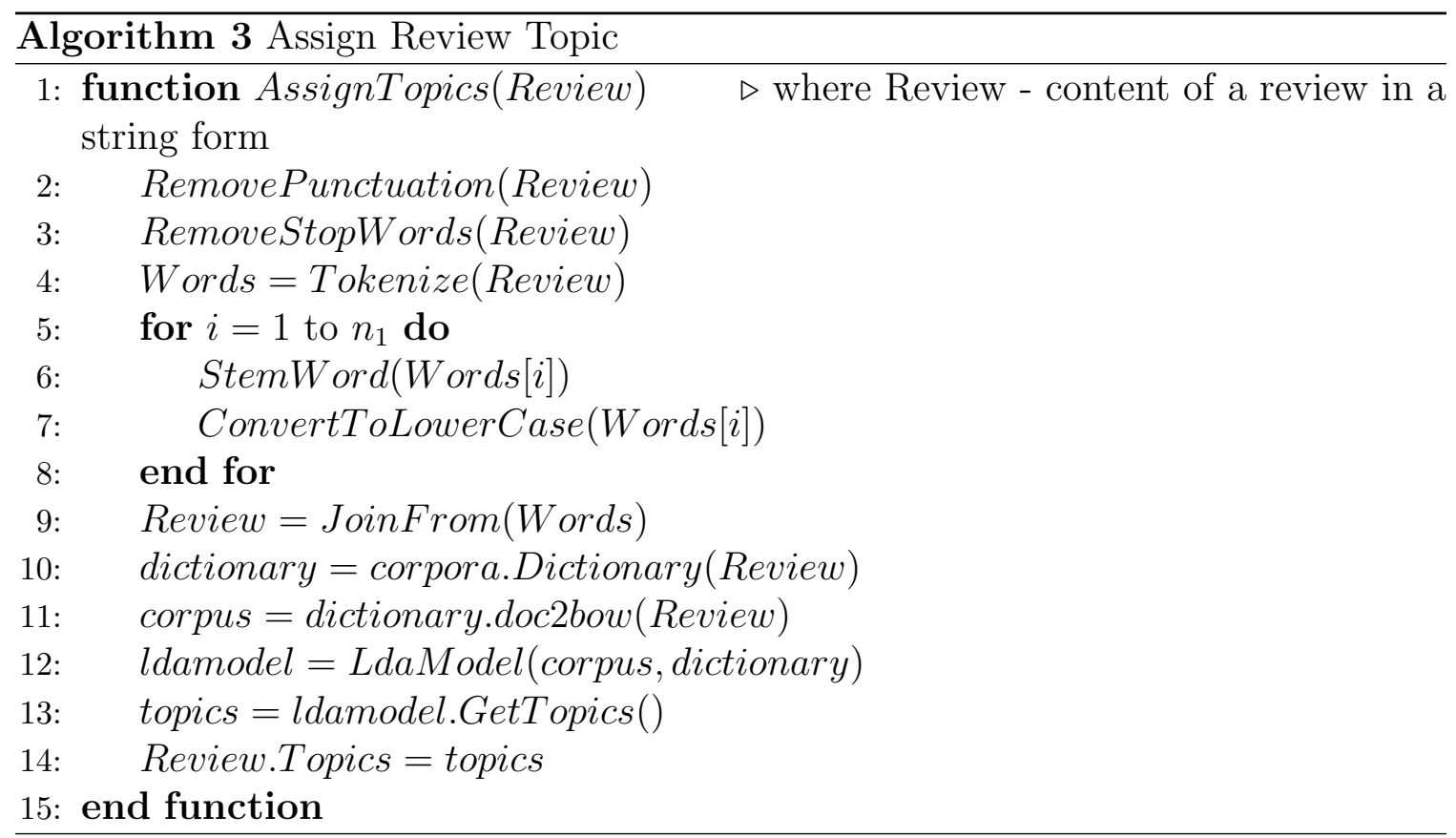

\subsection{Sentiment Analysis}

In order to gather more insights about each topic, we determine the sentiment of the reviews. The sentiment allows us to understand what attitude/feeling the user tried to express in his/her review. We believe that attaching sentiment to the user reviews can provide developers with additional key information on what topics are of negative sentiment (e.g., why users had negative experience) or what features or functionality of the app contribute to positive emotions.

Table 3.4: Sentiment categories.

\begin{tabular}{cc}
\hline Criteria & Assigned Label \\
\hline $\mid+$ Score $\mid>=3$ & Highly Positive \\
$\mid-$ Score $\mid>=3$ & Highly Negative \\
$\mid+$ Score $|>|-$ Score $\mid$ & Positive \\
$\mid-$ Score $|>|+$ Score $\mid$ & Negative \\
$\mid+$ Score $|=|-$ Score $\mid$ & Neutral \\
\hline
\end{tabular}


We used SentiStrength [39] for mining sentiment of user reviews. The SentiStrength library uses positive and negative words when determining the sentiment of a given sentence, where in our case the sentence is the complete review. SenthiStrength provides different results for sentiment: a binary result (positive and negative), a trinary score (positive, negative and neutral), or a single scale result (-4 to 4). We used the binary format and created our own labels based on the two scores provided by SentiStrength. Table 3.4 defines the mechanism we used for assigning sentiment labels to the reviews. For each review, we pass it through our sentiment algorithm 4 which uses the Senti-Strength library to get two separate scores (i.e., one for positive and the other for negative). Then using the Table 3.4 we check for both scores separately and assign one of the five categories (i.e., highly positive, highly negative, positive, negative, and neutral) to it.

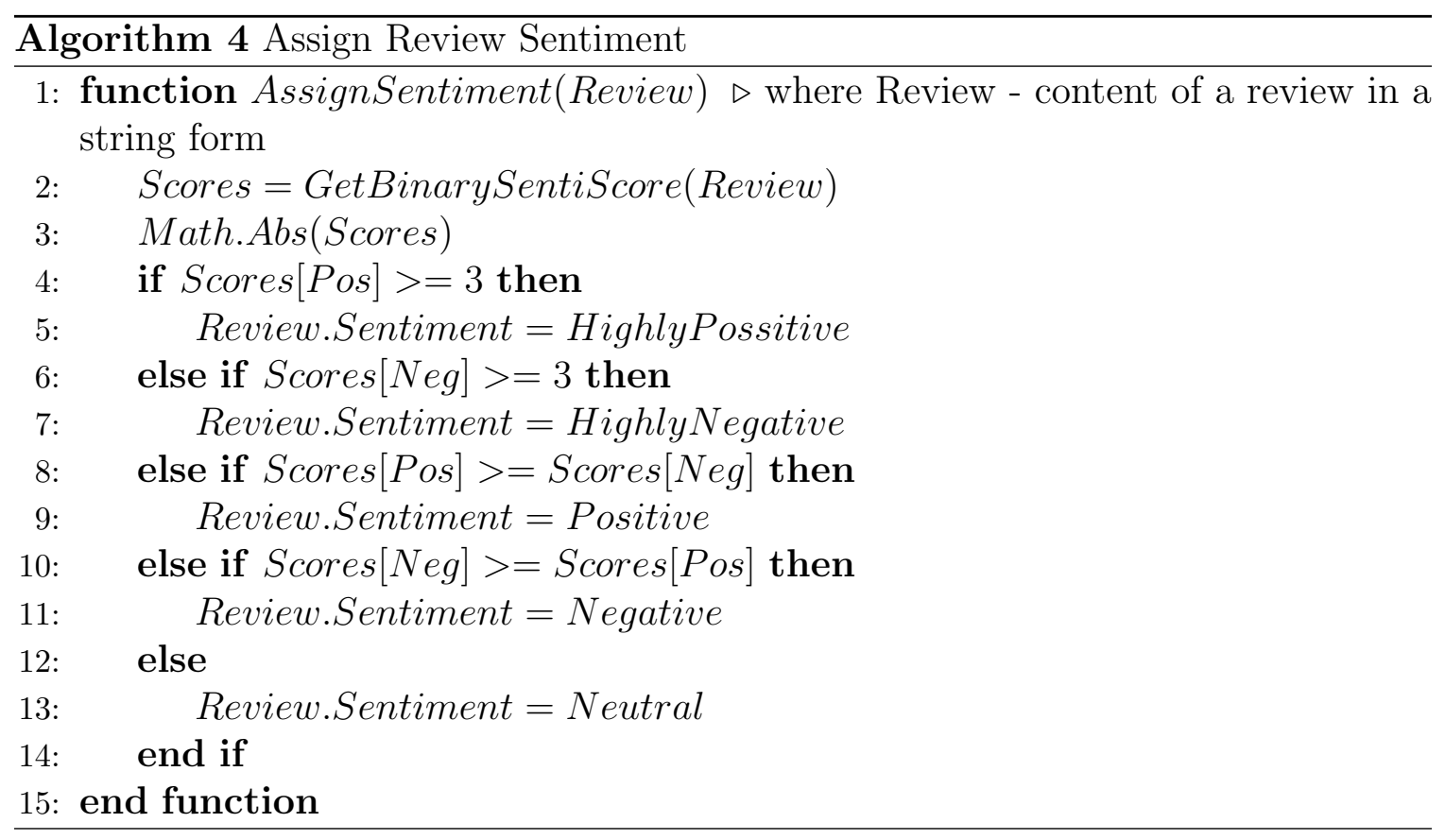

Algorithm 4 describes the complete process of assigning a sentiment label to the review. We utilize Table 3.4 to define the criteria as to how a review will be given a particular sentiment. We start off by passing our review (string) to the SentiStrength java library to calculate the sentiment and provide us with the binary score for it (line 2). This library call is provided by the SentiStrength documentation and is a simple 
one line console call. The result of this function will be two integer variables with keys Pos (representing the positive score) and Neg (representing the negative score). Since by default SentiStrength provides a negative value for the negative score, we simply calculate the absolute value (line 3) as we do not consider the sign. Afterwords, we have a simple criteria that we follow to assign the review one of the five sentiment labels (lines 4-14).

\subsection{Clustering Reviews}

To group related reviews together (e.g., those requesting the same new feature, discussing the same bug, or commenting on the same user experience), we cluster reviews based on the topics of the review along with the review title (i.e., the title provided by the user). We create a uni-gram based list of words for each review, as well as for all the reviews for a single app. We then assign to these lists a weight based on the TF-IDF measure [3]. This weight can be defined as follows:

$$
w_{i, j}=\frac{f_{i, j}}{\max _{z} f_{z, j}} \log \frac{N}{n_{i}},
$$

where $w_{i, j}$ is the weight for the word $\left(k_{i}\right)$ in the review $\left(d_{j}\right)$. The $f_{i, j}$ represents the frequency of the word $\left(k_{i}\right)$ in the review $\left(d_{j}\right)$. The max is calculated for all the words in the review. To decrease the weight for the words that appear in multiple reviews, we add the log function for the total number of reviews $(N)$ and in how many documents the word appeared $\left(n_{i}\right)$.

After calculating these weights, our review becomes a vector consisting of the combination of these weights. The review then is defined as follows:

$$
\operatorname{review}\left(d_{i}\right)=\left(w_{1 i}, w_{2 i}, w_{3 i}, w_{4 i} \ldots . w_{k i}\right)
$$

Now that we have represented all the reviews as a TF-IDF vector of the topics and title, we use the Vector Space (VS) [4] cosine similarity to identify the clusters of related reviews: 


$$
\cos \left(\overrightarrow{d_{1}}, \overrightarrow{d_{2}}\right)=\frac{\overrightarrow{d_{1}} \cdot \overrightarrow{d_{2}}}{\left\|\overrightarrow{d_{1}}\right\|_{2} *\left\|\overrightarrow{d_{2}}\right\|_{2}}
$$

Based on the cosine similarity, we group similar and related reviews together in a cluster.

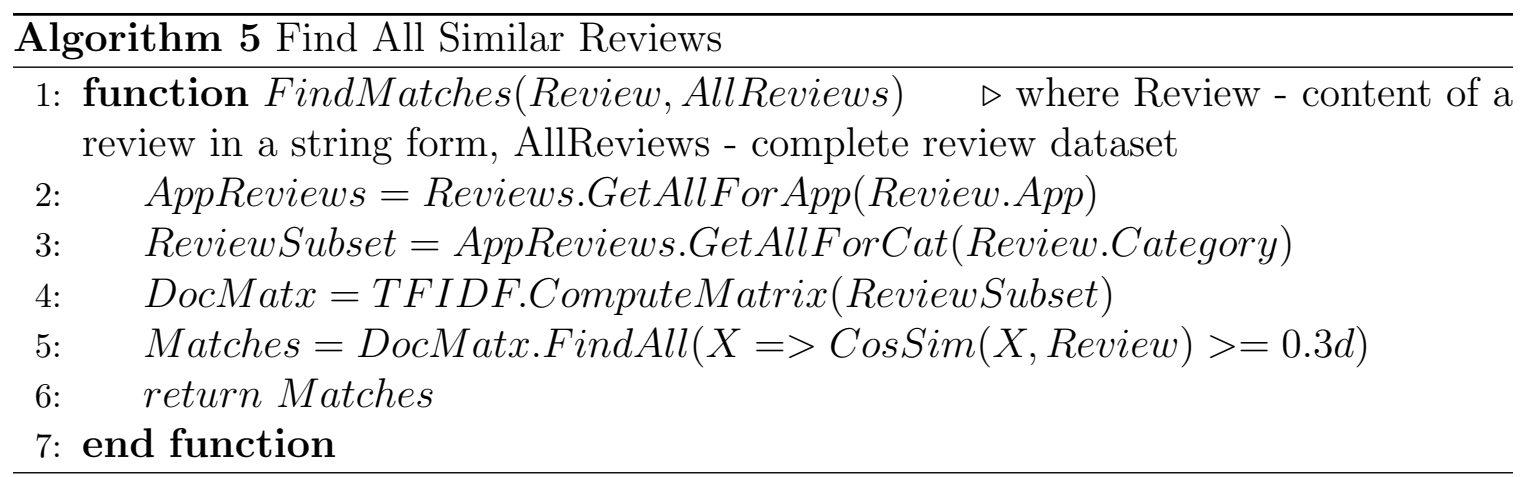

In Algorithm 5, we utilize TF-IDF to compute document matrices and find similar reviews. We start by filtering out the rest of the reviews. We only select reviews for the same app as our primary review (line 2), then from the reviews of the same app we select only the ones that are in the same review category (i.e, Bug Report, Feature Request, Rating, User Experience, or Other) (line 3). Now this ReviewSubset contains the list of all reviews for a particular App for a single Review Category. For this list we compute the TF-IDF vectors of all reviews and arrange them in a matrix (line 4). From this matrix we are able to identify the vector of a specific review. We considered two vectors to be similar if the cosine similarity among them was greater than 0.3 (line 5). This final output also contains the original review as the cosine similarity between itself will be 1 . This " 0.3 threshold" for similarity was determined by empirically evaluating multiple variations between 0 and 1 . We found that because the vectors were made with topics from LDA and not by the actual review, the " 0.3 threshold" was sufficient to determine whether the clusters are similar to each other. 


\subsection{Extracting Cluster Summaries}

Once we have identified the clusters of related reviews, we create a summary for each cluster. These summaries can help developers quickly understand what the users are trying to communicate with them. Each cluster may contain tens or hundreds of reviews. To ease developer comprehension of these reviews in each cluster, we extract a cluster summary consisting of the key sentences of all the reviews in that cluster. We used OTS [5] for generating cluster summaries.

OTS is an open source library for summarizing texts. It reads a text and decides which sentences are important and which are not. OTS creates a short summary or highlights the main ideas in the text. We used the summarization ratio of $30 \%$, meaning that the output file (i.e., cluster summary) is 30\% the length of the input file (all reviews within a cluster), based on the number of sentences in the input file. The recommended ratio for OTS according to their documentation is between 20 to 40 percent. We empirically determined (i.e., trough trial and error) that $30 \%$ worked best for the length of the summaries for reviews. Our understanding is that because the majority of the reviews are short texts, combining them together and compressing them at this ratio can yield better results. Anything above this would be repetition of previous text.

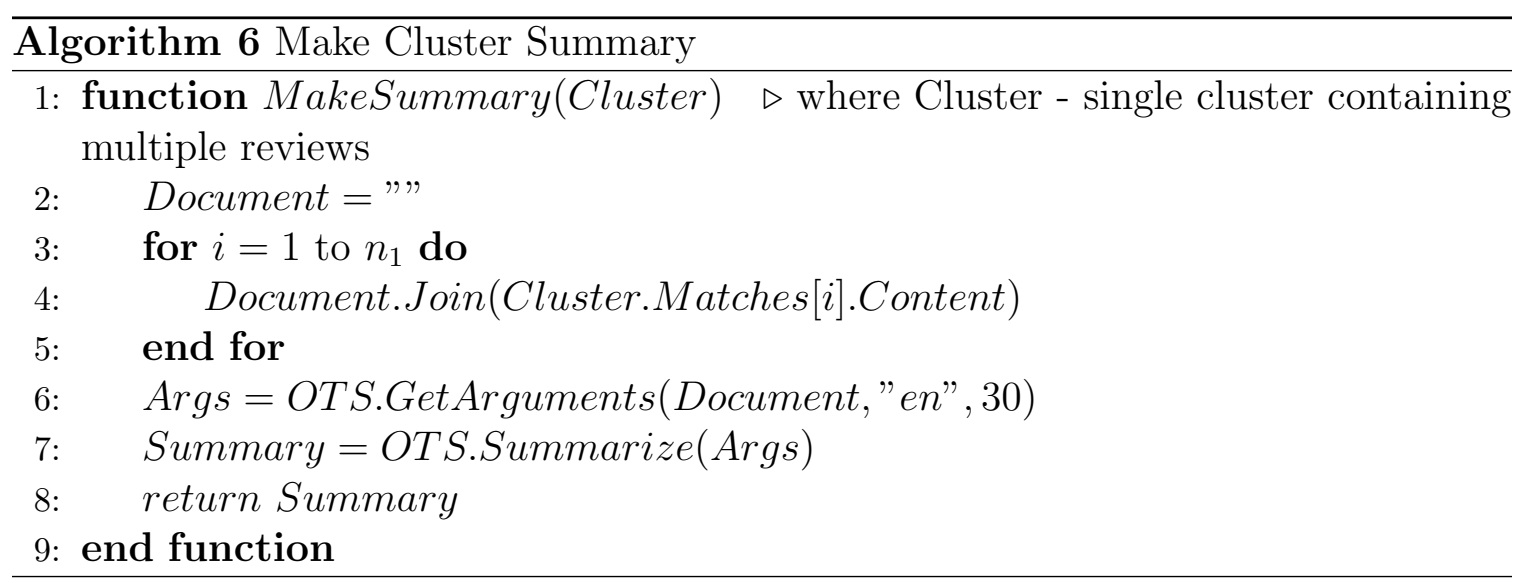

Algorithm 6 explains how we generated cluster's summaries. We first combine all the reviews within one cluster (lines 2-5). In order to use OTS Library, we need to create arguments that we will pass to summarize. In order to create these arguments, 
we need to provide the OTS library with a) the complete document with all reviews, b) the language of the document, and c) the percentage of output (line 6). We use a $30 \%$ length as for our dataset it had the optimal result based on our manual testing. Afterwords, the OTS library takes these arguments and generates a skimmed summary containing the most important sentences (line 7).

OTS works by creating a custom list of words from the provided document and sorting them according to how much they were used. As a result, OTS generates a sorted list of words based on how much it thinks they are important. OTS tries to find the sentences that are using these important words and only keeps those sentences, removing all others. Generated cluster summaries provide developers with the sentences containing only important words.

\subsection{Output of the Technique}

The results (i.e., output) of applying our technique of mining user reviews and leveraging insights from them is a set of clusters. Each cluster contains a title which is made of the topics of the reviews within it. This title is considered as a representation of what the users are talking about. We used a custom list of stop words to create these topics as the traditional English stop word dictionary ${ }^{3}$ was not efficient. This was due to the words that are not exactly classified as stop words but are too generic to be made as topics. We removed all such generic words regarding the apps and categories (e.g., app, game, play, ios, etc.) because they do not convey what the user is actually trying to say. Along with the title, the cluster contains a summary that consists of the most important sentences for all the review within the cluster.

The cluster also contains all the reviews within it with the details of each review including its own topic, user, content, ranking and so on; the category the cluster belongs to (bug report, feature request, user experience, rating, or other), and the overall sentiment of the cluster (positive, negative, etc.). The clusters will be ranked among each other based on the number of reviews and the overall sentiment of the cluster. The more the users talk about the same thing, the more important the cluster

\footnotetext{
${ }^{3}$ https://pypi.python.org/pypi/stop-words
} 
becomes.

\subsection{Data Analysis}

To answer our research questions we used various datasets depending on the context. Table 3.5 summarizes the number of apps and the number of reviews for each research question.

Table 3.5: Datasets used in research questions.

\begin{tabular}{cccl}
\hline RQ & \# Apps & \# Reviews & Selection of sample \\
\hline$R Q_{1}:$ & 20 & 1,000 & Randomly selected apps \\
$R Q_{2}:$ & 1 & 400 & One randomly selected app \\
$R Q_{3}:$ & 21 & 1,400 & Datasets used in $R Q_{1}$ and $R Q_{2}$ \\
$R Q_{4}:$ & 35 & 14,000 & Original dataset of 35 apps \\
\hline
\end{tabular}

To address our $R Q_{1}$, the author manually classified 1,000 user reviews (the same number of reviews were manually classified in [18]) into one of the five review categories such as bug report, feature request, user experience, rating, and other. These reviews were equally distributed among 20 randomly selected apps (from the original dataset of 35 apps). In total, 155 reviews were labelled as bug reports, 93 as feature request, 378 as rating, 212 as user experience, and 162 as other. The review distribution among our five categories was similar to the review distribution among three categories of [18] if our two newly introduced categories (i.e., "user experience" and "rating") were combined into "other" category.

For our $R Q_{2}$, we selected a small sample consisting of 400 reviews. These reviews were selected for a single randomly selected app (i.e., app called "Instasave — Grab from Instagram Photos and Repost It") from our original dataset. To evaluate the accuracy of the cluster generation step, we performed a manual inspection of the achieved results (400 reviews grouped into 182 clusters).

For answering $R Q_{3}$, we consider the datasets used in $R Q_{1}$ and $R Q_{2}$ for calculating and comparing the time it took us to perform the manual classification of reviews into five categories and the manual inspection of the generated clusters with the time 
of running our automated technique.

For $R Q_{4}$, we extracted hot topics and summaries for the complete dataset of 35 apps consisting of 14,000 reviews. However, to offer an example of the generated hot topics and their summaries, we consider the Instasave app used in $R Q_{2}$.

We evaluated the performance of our technique in terms of the recall, precision, F-measure and accuracy [3]. Precision is the fraction of relevant instances among the retrieved ones:

$$
\text { precision }=\frac{\mid \text { relevant_documents }|\cap| \text { retrieved_documents } \mid}{\mid \text { retrieved_documents } \mid},
$$

while recall is the fraction of relevant instances retrieved from the relevant ones:

$$
\text { recall }=\frac{\mid \text { relevant_documents }|\cap| \text { retrieved_documents } \mid}{\mid \text { relevant_documents } \mid} .
$$

Both of these measures take into account the relevance of the documents, while F-Measure is the harmonic mean of precision and recall:

$$
F-\text { Measure }=2 . \frac{\text { precision } . \text { recall }}{\text { precision }+ \text { recall }} .
$$

Accuracy is the degree of closeness of measurements of a quantity to that quantity's true value:

$$
\text { accuracy }=\frac{\mid \text { relevant_documents } \mid}{\mid \text { all_documents } \mid} .
$$




\section{Chapter 4}

\section{Results}

In this chapter, we present the results of our study by answering our research questions (Sections 4.1, 4.2, 4.3, and 4.4, respectively).

\section{1 $R Q_{1}$ : How accurate is our technique in classi- fying user reviews according to different cate- gories?}

To answer our first research question, we measure the accuracy our technique achieves in classifying user reviews into five categories. Figure 4.1 shows both the distribution of user reviews per each category, as well as distribution of the generated clusters (i.e., $R Q_{2}$ ) per each of the categories.

Instead of going through the user reviews, the developer can easily check the review clusters that summarize the major points of the reviews. We can observe that clustering reduces the effort of reading all the reviews by $41 \%$. This observation was concluded by matching the total number of reviews in the dataset with the total number of clusters. So even if the developer went on with reading all of the clusters he/she would still have saved about $41 \%$ of the work effort.

For calculating the accuracy of our technique (as mentioned in Chapter 3.9), we used a subset of our data (sample) and manually classified it into the five categories 
Table 4.1: Examples of classifying user reviews.

\begin{tabular}{|c|c|c|c|c|}
\hline App Name & Review & Topic & Category & Sentiment \\
\hline \multicolumn{5}{|l|}{ StarMap 3D+ } \\
\hline & $\begin{array}{l}\text { I can't say enough } \\
\text { about this app! }\end{array}$ & app cant say enough & Bug Report & Neutral \\
\hline & $\begin{array}{l}\text { Amazing app, one } \\
\text { suggestion though. } \\
\text { You guys should add } \\
\text { exoplanets! }\end{array}$ & guy one exoplanet though add & Feature request & HighlyPositive \\
\hline & $\begin{array}{l}\text { Compass is reversed. } \\
\text { Help! }\end{array}$ & revers help compass & User experience & Neutral \\
\hline & COOL! & cool & Rating & Positive \\
\hline & 0̆09du008f & [no topic] & Other & Neutral \\
\hline \multicolumn{5}{|l|}{ Algebra Touch } \\
\hline & $\begin{array}{l}\text { Nothing works, } \\
\text { crashes every time. I } \\
\text { want my } 2.00 \text { back, I } \\
\text { DEMAND IT!!! }\end{array}$ & time back crash demand want & Bug report & Neutral \\
\hline & $\begin{array}{l}\text { Just wish there were } \\
\text { more levels. }\end{array}$ & wish just level & Feature request & Neutral \\
\hline & Great help & great help & User experience & HighlyPositive \\
\hline & Great & great & Rating & HighlyPositive \\
\hline & More! & [no topic] & Other & Neutral \\
\hline \multicolumn{5}{|c|}{ Babbel Learn 14 languages } \\
\hline & $\begin{array}{l}\text { Mic input doesn't } \\
\text { work most of the } \\
\text { time. }\end{array}$ & doesnt input mic work time & Bug report & Neutral \\
\hline & Please add Japanese & pleas add japanes & Feature request & Positive \\
\hline & $\begin{array}{l}\text { Needing to learn } \\
\text { Finnish! }\end{array}$ & need finnish learn & User experience & Neutral \\
\hline & Good app! & app good & Rating & Positive \\
\hline & Deleting! & delet & Other & Neutral \\
\hline
\end{tabular}

(bug report, new feature request, user experience, rating, and others). This sample data contained a total of 1,000 reviews of 20 randomly selected apps. Table 4.2 reports the recall, the precision, the F-Measure for each category, as well as the overall accuracy achieved by our technique when classifying user reviews. User experience is the most misclassified category of reviews. We found that the majority of these reviews were assigned to the rating category.

We achieved the overall accuracy of $81.5 \%$. Previous work [18], has reported the overall accuracy of $86 \%$ when classifying reviews into three categories. With the increase of the number of categories, the accuracy of the classification process tends to decrease. We believe our technique of classifying user reviews is promising since 


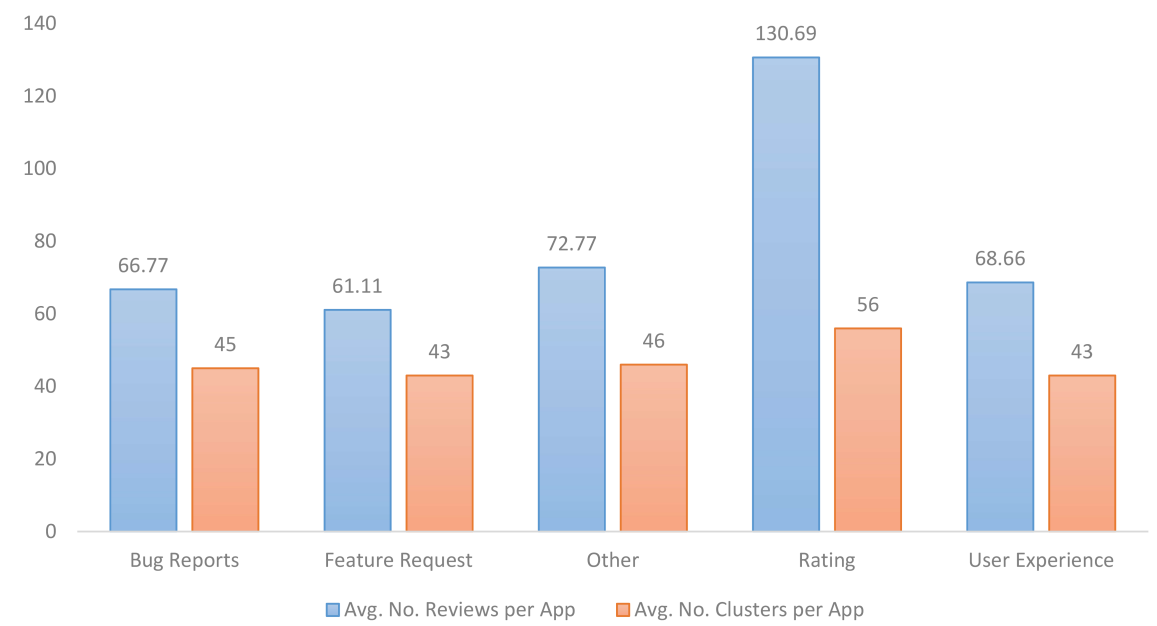

Figure 4.1: Distribution of reviews vs. distribution of clusters per category.

we classify user reviews into five different categories.

In Table 4.1, we report several examples of the results achieved by the review classification step. Due to the lack of space, we select three random apps (rest of the results are presented in Appendix A) from our dataset and five random reviews for each of these apps (i.e., one review for each category). Table 4.1 reports the topic, the category and the sentiment label our technique assigned to each review. Topics here define the most important words within a review. In some cases (i.e., the last review for the first and second app), we can see that the LDA could not assign any topic because of the inadequate (poor quality) nature of the review.

We have also included detailed results for all our selected 35 apps in the Appendix A. Table A.1 reports one review per review category for each app. We can see that most of the reviews in the other category are being presented as empty because those reviews contained characters from a different language that our technique was unable to understand. This usually occurs when the customer is using a nonQWERTY keyboard. Since our LDA stemmed all the words, the final words in the topic are already stemmed. We also found out that a lot of users use a short message format when writing reviews and often misspell words which cause our algorithm to sometime classify the reviews incorrectly.

Comparing our accuracy with the CLAP tool [18], we achieve the accuracy is $82 \%$ 
as apposed to the CLAP's 86\%. However, when comparing F-Measure, our value of $80.12 \%$ is only $2.5 \%$ away from the CLAP's $82.66 \%$ which is significant given that we classify reviews into five categories (unlike three categories in their work).

$R Q_{1}$ : Our technique achieves the overall accuracy of $81.5 \%$ when classifying user reviews into five categories.

Table 4.2: Classification accuracy of user reviews.

\begin{tabular}{ccccc}
\hline Category & \# Reviews & Precision & Recall & F-Measure \\
\hline Bug report & 155 & 76.97 & 88.39 & 82.29 \\
Feature request & 93 & 65.19 & 94.62 & 77.20 \\
Rating & 378 & 94.46 & 81.22 & 87.34 \\
User experience & 212 & 90.73 & 64.62 & 75.48 \\
Other & 162 & 69.19 & 90.12 & 78.28 \\
\hline Overall & 1,000 & 79.31 & 83.79 & 80.12 \\
\hline \multicolumn{5}{c}{ Overall Accuracy : $\mathbf{8 1 . 5 \%}$} \\
\hline
\end{tabular}

\section{2 $R Q_{2}:$ How accurate are the review clusters generated by our technique?}

Since we are interested in supporting developers in their release planning and maintenance tasks, the only interesting categories would be bug report, feature request and user experience. As reported in Figure 4.2, we found that these three categories contain on average $56 \%$ of the clusters per app. Through our manual inspection we observed that majority of the failures lie where the review is missclassified from one of these three categories to rating.

In terms of the sentiment, overall (note that not all of the results are reported here), we observed that when users report bugs and problems, their sentiment tends to be more negative about the app making the overall review being categorized as negative. When users are requesting features or enhancements to improve their user 


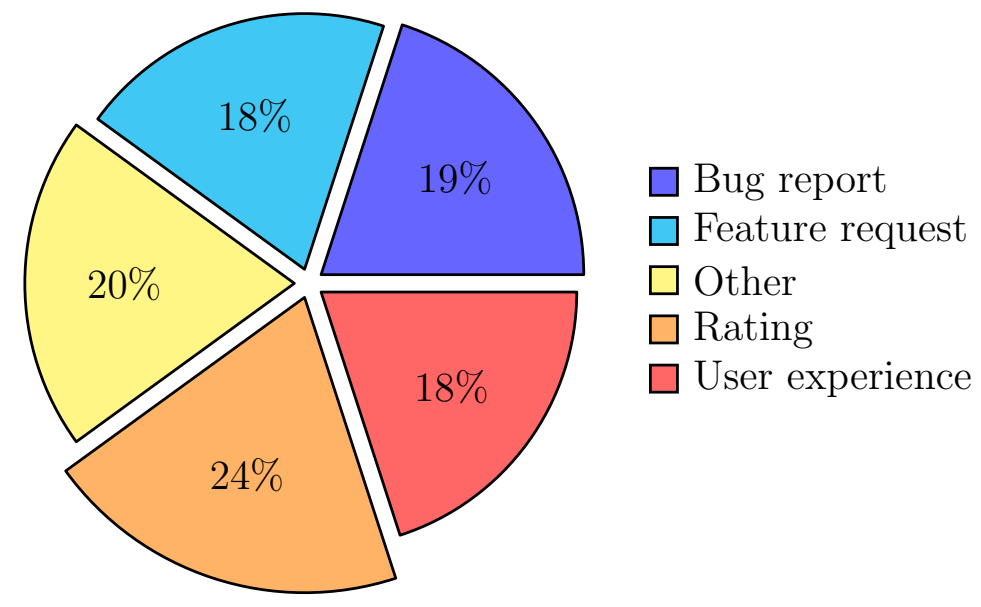

Figure 4.2: Distribution of clusters.

experience, their reviews tend to give a sense of likeness for the app and their sentiment tends to be on the positive side. Another interesting observation is that if the app has a negative rating, the developer can quickly go through the top clusters within the rating category to determine the issue with the app. This would be the key benefit for the developer if she notices a drastic change in the ratings of a recent version.

As explained in Chapter 3.9, we evaluated the accuracy of the cluster generation step based on the results achieved for a single app (i.e., "Instasave"). Our technique generates and summarizes all the reviews in our dataset for the app (i.e., 400) into 182 clusters. It takes about 14.37 minutes for our technique to run; this time includes the categorization, topic modelling, sentiment analysis and cluster generation steps. We achieved the overall accuracy of $78.67 \%$ for the cluster generation step. This indicates how well our technique is able to leverage the information from the reviews. We observed that the top clusters generated in this step represent all the issues that require instant attention from the developer.

Table 4.3 reports the results we achieved from the manual inspection of the clusters. We found that the most frequent misclassification error in this step belongs to the case where the technique generates multiple clusters for the same kind of reviews. Our technique fails to merge these reviews because of the presence of either a single 
Table 4.3: Accuracy for cluster generation (single app study).

\begin{tabular}{cccc}
\hline \multicolumn{4}{c}{ App Name : "Instasave" } \\
\hline Category & \# Clusters & Avg. \# Reviews & Accuracy \\
\hline Bug report & 30 & 2.24 & 74.44 \\
Feature request & 23 & 1.65 & 78.15 \\
Rating & 58 & 3.51 & 83.21 \\
User experience & 24 & 3.51 & 75.07 \\
Other & 47 & 1.17 & 82.37 \\
\hline Overall & 182 & 2.19 & 78.67 \\
\hline
\end{tabular}

unique topic or a difference in the titles for the reviews. We found a 1 to 2.2 ratio between a cluster and the number of reviews for this particular app. We re-ran our technique on a few other apps and found a similar ratio of the cluster and review numbers.

Comparing our accuracy with CLAP [18], we found that our cluster accuracy for bug reports is 74.44, a percentage higher than CLAP's 73\%. However, CLAP's accuracy for clustering feature requests of $87 \%$ is much higher than our accuracy of $78.15 \%$. This is due to the difference in the multi-class classifications.

$R Q_{2}$ : Our technique achieves the overall accuracy of $78.67 \%$ when creating and summarizing clusters.

\section{3 $R Q_{3}$ : How much time, on average, does our technique save when compared to a manual process of review-based development planning?}

A typical scenario is that developers are manually going through users reviews trying to understand the overall user feedback on their apps. Due to volume and velocity of the user reviews, keeping track of the user requirements can be a tedious task. In order for us to compare the time of the manual process of leveraging insights from 
user reviews with the time of automating the process, we calculate how much time the developer would save as opposed to some trade off for accuracy. Even though the algorithm had an overall accuracy of about $81.5 \%$ in classification of reviews and of $78.7 \%$ in clustering, we found that it was promising enough in finding the most important and most "talked about" issues.

To calculate the time, we considered the same datasets used for $R Q_{1}$ (Chapter 4.1) and $R Q_{2}$ (Chapter 4.2). In terms of the manual effort, it took us 6.5 Person Hours $(\mathrm{PH})$ to correctly classify the 1,000 reviews, while the technique was able to do it in 3.6 seconds. For the $R Q_{2}$, the manual process of cluster generation was such a time consuming task that we opted for a manual inspection of clusters instead. The manual inspection of clusters took about $3 \mathrm{PH}$. It took 14.37 minutes for the technique to generate clusters. The most time is saved by using LDA for generating topics and the Vector Space Model.

$R Q_{3}$ : Automating the process of leveraging user insights from the user reviews can save several hours (on average, 10.8 hours) compared to the manual process.

\section{4 $R Q_{4}$ : What are the hot topics that users talk about?}

One of the most useful for developers insights from the user reviews are the hot topics that the users are talking about. The main challenge that developers face here is to be able to quickly identify those problems or bugs that are reported by several users via reviews. Therefore, our goal is to help developers quickly scan all the reviews and summarize the major issues discussed by the users. Our technique can quickly determine hot topics discussed in the reviews and summarize these reviews to allow developers identify major issues and plan for the next release.

To extract hot topics and their summaries, we ran our technique on the complete dataset consisting of 14,000 reviews for 35 apps. The dataset to be shared with this study reports these results. However, for the sake of demonstrating the generated hot 
topics and their summaries, we select the same app used in answering our $R Q_{2}$ (i.e., Instasave - Grab from Instagram Photos and Repost It) and show one hot topic (i.e., major cluster) for each category.

We applied our technique on the complete dataset to find out the various topics that the users were talking about. We found various results for clusters. We plotted them using an informative tree. Figure 4.3 show a sample part of that tree for just one app in the "Music" category. We can see the various categories for reviews and the most hot cluster within that category. We have added more detailed Figures B.1, B.2, B.3, B.4 in the Appendix B. Those figures provide a more detailed distribution of the results. We have various ratios on top of the categories that report how many, in total, clusters were present in that category for a certain app and how many are shown in the figure (e.g., 1/49). We presented one app per category and for each app we presented one cluster per review category. We also have included summaries attached in those figures. For majority of the clusters we can see that the summaries report a complete aspect of the user's opinion.

Table 4.4 reports the hot topic (major cluster) for each category, the number of reviews, the generated summary (i.e., most important sentences across all reviews), and the sentiment labels for the Instasave app. For example, for the bug report category our technique identifies a hot topic represented by the issue that was reported by six different users where they could not start the application because of an error. Our technique is able to calculate the similarities among the reviews and determine whether they are discussing the same issue.

Summary of the hot topic presents the most important sentences of all the reviews for that topic (cluster). By just reading the summary of each cluster, the developer can quickly scan through the key sentences and understand what the users concerns are (e.g., an error with code 403 for the bug report's topic summary). 


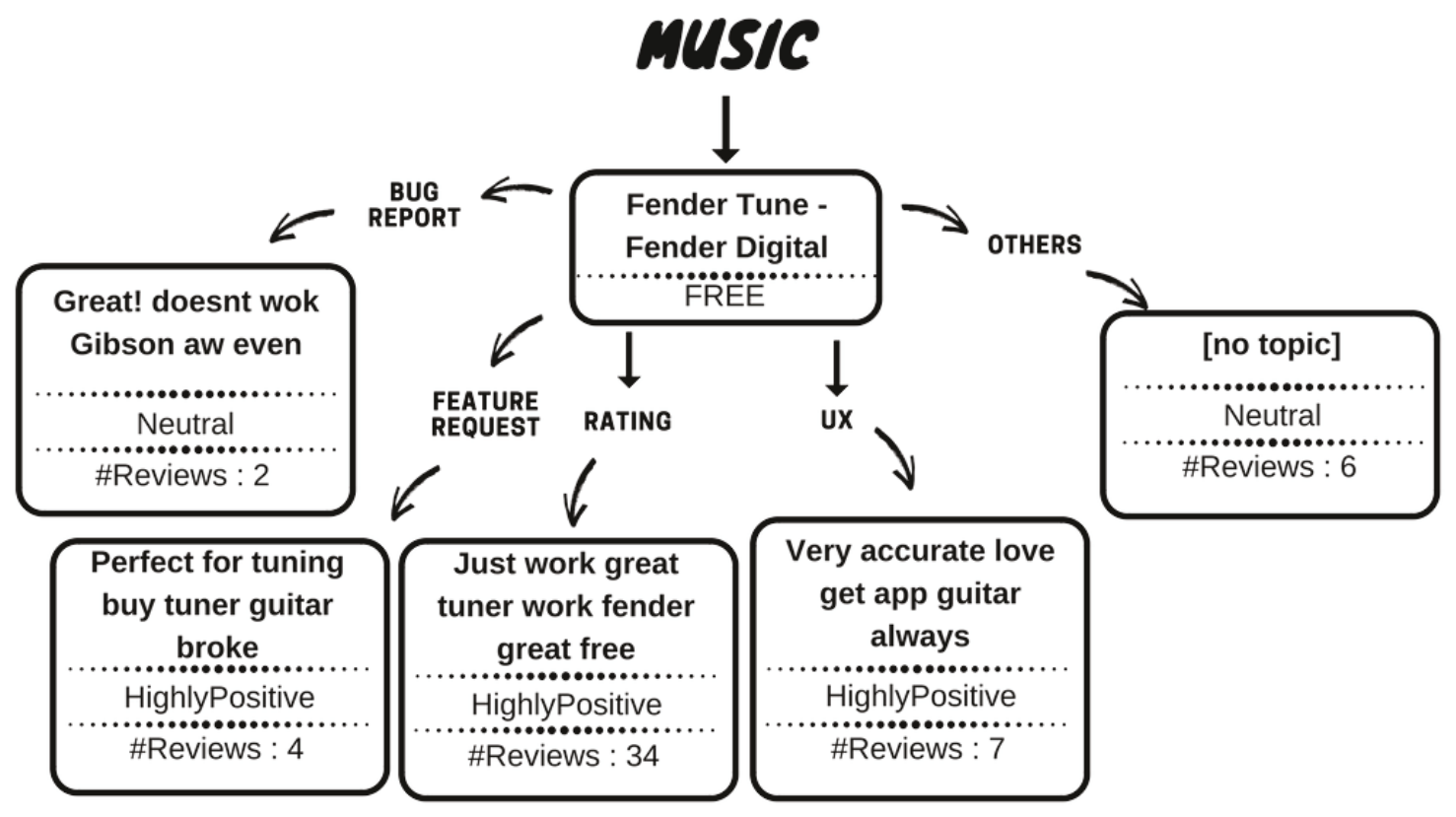

Figure 4.3: Sample cluster results.

The sentiment column reports the overall sentiment for the cluster. Analyzing and presenting the sentiment for the whole table, our technique allows the developer to determine that the app overall generates positive feedback from the users. While for the Instasave app, the top cluster for user experience reports a positive experience, we found that for some apps the user experience of the negative sentiment is associated with the users' misunderstanding of the app functionality (e.g., the correct use of a feature).

$R Q_{4}$ : Each app may have several hot topics within each category of user reviews. These hot topics and their summaries allow developers to identify key themes users talk about, either of positive or negative sentiment. 
Table 4.4: Hot topics and summarization.

\begin{tabular}{|c|c|c|c|c|c|}
\hline \multicolumn{6}{|c|}{ App Name : Instasave - Grab from Instagram Photos and Repost It } \\
\hline ClusterID & $\begin{array}{c}\text { Hot Topic } \\
\text { (Cluster } \\
\text { Title) }\end{array}$ & Category & \# Reviews & Summary & Sentiment \\
\hline 0 & $\begin{array}{l}\text { App will not } \\
\text { open and I } \\
\text { get an error } \\
\text { message. app } \\
\text { user client exist } \\
\text { sandbox }\end{array}$ & Bug report & 6 & $\begin{array}{l}\text { Every time I try to open } \\
\text { the app, I get this mes- } \\
\text { sage, "code": 403, "er- } \\
\text { ror type": "OAuthForbid- } \\
\text { denException", "error mes- } \\
\text { sage": "You are not a sand- } \\
\text { box user of this client". }\end{array}$ & Negative \\
\hline 1 & $\begin{array}{l}\text { Great want } \\
\text { video save } \\
\text { opportun love }\end{array}$ & Feature request & 4 & $\begin{array}{l}\text { THIS IS MY GO TO } \\
\text { APP WHEN WANTING } \\
\text { TO DOWNLOAD VIDEOS } \\
\text { FROM INSTAGRAM! Re- } \\
\text { ally like this app makes it } \\
\text { easy to download vids you } \\
\text { want in your camera roll. }\end{array}$ & HighlyPositive \\
\hline 2 & Amazing help & User experience & 7 & $\begin{array}{l}\text { This is so helpful It let you } \\
\text { download the photo directly } \\
\text { to camera roll without copy- } \\
\text { ing and pasting the link like } \\
\text { other apps, and you can re- } \\
\text { move ads for free. }\end{array}$ & HighlyPositive \\
\hline 3 & Love it great app & Rating & 56 & $\begin{array}{l}\text { Great with downloading } \\
\text { videos Great app' Great } \\
\text { app Great love it really } \\
\text { really love this app I love } \\
\text { it Great app, really nice to } \\
\text { save videos Great It's great } \\
\text { for reposting all the videos } \\
\text { I like and wanna share with } \\
\text { my followers and convient } \\
\text { Great Great app This is } \\
\text { awesome This is the best } \\
\text { ever I love this app It's a } \\
\text { great app that I use more } \\
\text { than any other downloading } \\
\text { app its great Great app. I } \\
\text { love it it's great i love this } \\
\text { app i love this app sm fam } \\
\text { I really love this app Love } \\
\text { this app Great app use it } \\
\text { all the time I love this app } \\
\text { it lets you save unlimited. }\end{array}$ & HighlyPositive \\
\hline 4 & $\begin{array}{l}\text { Awesome use } \\
\text { suppos easi }\end{array}$ & Other & 8 & $\begin{array}{l}\text { Whether you are new to In- } \\
\text { stagram or not this app will } \\
\text { boost up your account for } \\
\text { sure Yes I give this app } 5 \\
\text { stars. }\end{array}$ & Neutral \\
\hline
\end{tabular}




\section{Chapter 5}

\section{Discussion}

In this chapter, we discuss our results and compare them with the findings of the previous studies (Section 5.1). We also discuss various implications of our technique for developers (Section 5.2), industrial practitioners (Section 5.3) and future researchers (Section 5.4). We describe threats to the validity of this study in Section 5.5.

\subsection{Comparative Analysis}

Villarroel et al. [18] in 2016 presented a tool for the Google Play store that uses the reviews exported by the developer to find useful information about their app. They focused on classifying the reviews in three categories (i.e., bug report, feature request, and others). They used machine learning approaches to train and classify the reviews into these categories. Then they created them into clusters based on the TF-IDF model. We used a slightly different approach when clustering and went for a keyword-based approach originally proposed by Maalej et al. [27]. We found that for the app reviews on the Apple's store categorizing them into five categories yield an average accuracy of $81.5 \%$, while the previous study [18] had an average accuracy of $86 \%$ when classifying for just three classes. Despite the fact that our classes are different from theirs when compared on the F-Measure the difference was 2.5\%.

For the end result comparison, we selected the two prominent research studies [18, $20]$ from the past that were most relevant to our work. Our system created hot topics 
based on the five review categories. Our goal was to automatically determine the most hot topic from a particular category to support app developers with better understanding of the users' feedback. Both of these previous studies had a similar objective, however they employed different approaches for identifying such clusters and used different datasets for evaluation. Because of these factors, it was difficult for us to develop a proper baseline for evaluation as we could not make one-toone comparison of the techniques. We achieved an average accuracy of $78.67 \%$ for clustering, while Villarroel et al. [18] achieved an accuracy of around $80 \%$ for their classes. Chen et al.[20], on the other hand, performed a binary class classification, where a review was either informative or not. They used a very different approach for grouping and computed a probability matrix for each review with a probability of how good the prediction of a review in a specific cluster is. They achieved an F-Measure of $87.7 \%$.

\subsection{Implications for Developers}

The presented technique provides a better way for the developers to get an understanding of the hot topics that their customers are talking about as apposed to going through them manually. This can help developers to quickly go through some of the reviews that are grouped into a cluster and understand the issues the users are facing. The technique is capable of taking just the Apple's App ID which is available to the developers and creating a complete report of hot clusters for that app's reviews. There are also capabilities within the technique to allow developers to only target a specific country and find the hot topics in that area. This can help find domestic issues that might only be present in some counties and not in the others. The most common example of such domestic issue is the lag within server-client communication for multiplayer apps. This happens when the server is located in a specific country, but because of a low ping within the server and the user base being within that country there is a lack of reviews discussing such issues. However, if one looks at the reviews from a different country, it becomes clear that the server needs to be optimized. 
There are various other implications for developers using our technique. We have found that this not only allows developers to better plan their future releases, it can also help them understand the overall user experience of the application through the User Experience (UX) and rating review category. If majority of the clusters within the review category are negative, then, there might be some misleading functionality that the user expected to perform differently. In some cases, we observed that an overall negative sentiment exists due to the app being a paid one and the price does not reflect the functionality it comes with. Users might be disappointed when it comes to the available features and the cost of the app. Our technique can help developers determine the optimal price range for their apps based on the users' satisfaction.

We also observed that in a development team different review categories can help different team members working on different part of the app. For example the graphical design might be more interesting in knowing the hot topics within the UX category. While the back-end developers would want to focus more on the bugs and their fixes. The team lead would be interested more in feature requests to have more users engaged with the app. Support team can analyze the "rating" category to understand what should be addressed next.

\subsection{Industrial Implications}

Due to the fast growing community in mobile app development, the focus of the majority of the companies have switched towards the mobile users. In order to create a user-focused development environment, companies can utilize our technique towards the development of a self organizing online review management portal. Due to how we have utilized the Apple's RSS feed the only input required is the iOS app's unique ID which is an ID provided by Apple to every developer for each app. Through this ID the portal can fetch all details regarding the app including the most recent reviews throughout the world. The portal will be helpful for all app developers to see what the major issues for their app are.

Another implication for industry can be a completely automatic management of reviews. As of now, our technique can automatically organize and cluster reviews 
into various categories. But after that, it requires the developer to understand what should be done with those clusters. This can be easily fixed by implementing an algorithm that takes these clusters and based on some requirement (e.g., number of reviews, review category) pushes the cluster directly to the development Git portal as an Issue or enhancement. This can help incorporate the user's opinion directly into the development environment.

\subsection{Implications for Future Research}

There have been numerous developments in the field of mobile application due to the latest trend of people preferring mobile devices over their computers for their day to day usage. Major research have been done recently focused on mobile application $[19,29,26,18,45]$. The major focus of these studies have been towards automating and improving the process of development in some way. However, only a few studies focused on a completely automated solution where the opinions were directly integrated with the development environment [17, 25, 24]. There is room to find better ways of integration and extraction of useful information from the app store data.

Developers try to find various ways for app store optimization that can help their application rank higher on search engines and help them show their app to a larger user base. Due to the latest advancements a simple keyword-based optimization approach is no longer valid. This is due to the fact that search engines have become more intelligent. Their ranking is based on the content and not the keywords. Therefore, there is some room for future research for using app store reviews in finding useful content topics for app store optimization. This would help developers find topics that users would be searching for and help them make content for their app for solving user's problems and in the end improving their search ranking.

LDA, on the other hand, is a more generic approach. It is usually used for article recommendation systems where researchers have trained the model on the Wikipedia dataset. This is a valid approach for making generic topics, however, for this research using a more domain-specific dataset would have improved the results. Development of such a dataset, more focused on mobile app development and the issues users face 
in mobile application, would be of a great advancement and help for the community. This would open new doors for researchers in improving existing algorithms and their performance.

\subsection{Threats to Validity}

Threats to internal validity are related to the quality of study design and rigorousness of its execution. In our work, these threats are related to the data collection, manual classification performed for $R Q_{1}$ and $R Q_{2}$, as well as data analysis.

Our dataset of user reviews was collected using Apple's RSS feed which places certain restrictions on the data requests and number of items per query (as discussed in Chapter 3.1). Regarding our dataset, in order for us to collect a representative sample of user reviews per app, we relied on a sampling formula (see Equation 3.1) that determines the right size of our sample. We followed robust app and review selection procedures and believe that we evaluated our technique on a relatively large and diverse set of apps and reviews.

Personal bias Since our manual inspection of the quality of the generated review categories and clusters was performed by a single researcher, we are aware of the bias that may have been introduced by the coder. This problem can also be observed in the app store selection. Another threat may be related to the choice of the clustering algorithm. We have applied techniques and tools (LDA, VSM, etc.) that are widely adopted by the software engineering research community.

External validity concerns the generalization of our findings. In our study, we focused on the user reviews of the apps hosted on Apple's App store, therefore, our findings are limited to the iOS apps. It is possible that our results would not generalize to other apps developed for other platforms such as Android or Windows phone. Our findings are limited to the reviews written in English. While our technique can work with the reviews written in other languages, it needs to be correctly adapted to work well for non-English-language texts. Another thread is that because we fetched the most recent reviews from the Apple's RSS Feed there is a possibility that some of these reviews might have been considered as spam. However, Apple 
has set a reliable security system and a specialized team for moderating any spam. Every review submitted by a user is passed through this system to ensure the quality standards are met. Based on our own manual inspection, none of the reviews were considered as spam or falsely placed to favour any review category. 


\section{Chapter 6}

\section{Conclusions}

The main goal of this thesis was: to automate the process of gathering and summarizing user's opinion from the bundle of reviews out there. To achieve this goal, we have implemented a technique that extracts the hot topics from the bundle of reviews and summarizes them to provide the developers the essence of what their user-base is talking about and we evaluated it by addressing a number of research questions. Section 6.1 presents a summary of our technique and the achieved results. Section 6.2 outlines possible future directions.

\subsection{Summary of the Results}

App developers face a challenge of leveraging insights from user reviews, e.g., understanding what bugs and problems users experience or what new features they desire. Manual inspection of the reviews, while possible, can be time consuming. But consid-

ering the volume and the velocity of user feedback, this manual process may not be feasible. Yet, understanding user feedback is essential for maintaining apps and planning their future releases. Therefore, automating the process of mining user reviews can help developers focus their time and efforts on what is important.

In this thesis, we introduced a technique that assists developers by automating the process of extracting useful information from user reviews. The technique first 
classifies user reviews into five categories. We then employ topic modelling for generating topics for the reviews, perform sentiment analysis, and group similar reviews into clusters. For each generated cluster we extract a summary by applying an open text summarization technique. Finally, the results are clustered according to the hot topics.

We performed an evaluation of various steps of our technique by addressing four research questions. We found that our technique is promising in classifying user reviews into five categories (bug report, feature request, user experience, rating and other) with the overall accuracy of $81.5 \%$, as well as generating clusters of related reviews with the overall accuracy of $78.67 \%$. Our technique is able to save several person-hours compared to the manual process. And finally, generated hot topics, their summaries along with the sentiment can be help developers quickly understand major points discussed by the users.

\subsection{Future Work}

We now discuss several future directions that can be followed to improve our work. First, addressing the problem of not being able to find all of the clusters that correspond to the topics. This can be done by analyzing the "rating" and "other" category of reviews and finding a better set of keywords that either improves the current multiclass classification or adds a new class to it. Second, we can improve our technique to be able to generate more accurate topics from short reviews that do not contain much information. And last, we used open text summarizer for generating summaries of reviews for each cluster. If there are too many reviews in a cluster, the summary can be quite lengthy. In future, we can apply a better summarization algorithm, perhaps using machine learning techniques to provide developers with shorter and more precise summaries. 


\section{Appendix A}

\section{Tables}

Table A.1: Sample app reviews.

\begin{tabular}{|c|c|c|c|c|}
\hline App Name & Review & Topic & Category & Sentiment \\
\hline \multicolumn{5}{|l|}{ Instasave } \\
\hline & It doesn't work & doesnt work & BugReport & Neutral \\
\hline & This works great you want photos get this! & $\begin{array}{l}\text { photo great work want } \\
\text { get }\end{array}$ & FeatureRequest & HighlyPositive \\
\hline & Very Helpful & help & UserExperience & Neutral \\
\hline & awesome & awesom & Rating & HighlyPositive \\
\hline & 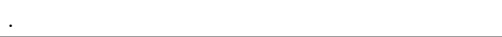 & no topic & Other & Neutral \\
\hline \multicolumn{5}{|l|}{$\begin{array}{l}\text { Saavn Music } \\
\text { and Radio }\end{array}$} \\
\hline & Solve it pls & solv pl & BugReport & Neutral \\
\hline & $\begin{array}{l}\text { Please make the new update compatible for } \\
\text { ipod } 4\end{array}$ & $\begin{array}{l}\text { pleas compat make new } \\
\text { ipod }\end{array}$ & FeatureRequest & Positive \\
\hline & Needs to be available on iOS 6.1 & need avail io & UserExperience & Neutral \\
\hline & Superb & superb & Rating & Neutral \\
\hline & Cheating & cheat & Other & HighlyNegative \\
\hline \multicolumn{5}{|l|}{$\begin{array}{l}\text { Followers So- } \\
\text { cial Analytics }\end{array}$} \\
\hline & Does everything. No glitches & glitch everyth & BugReport & Neutral \\
\hline & Would recommend & recommend & FeatureRequest & Neutral \\
\hline & Gets the job done & job done get & UserExperience & Neutral \\
\hline & Good & good & Rating & Positive \\
\hline & Mnnnj & mnnnj & Other & Neutral \\
\hline \multicolumn{5}{|l|}{$\begin{array}{l}\text { Add Back- } \\
\text { ground Music } \\
\text { To Video }\end{array}$} \\
\hline & This crap doesn't work & doesnt work crap & BugReport & HighlyNegative \\
\hline & wish it had more music variety though & $\begin{array}{l}\text { wish varieti music } \\
\text { though }\end{array}$ & FeatureRequest & Neutral \\
\hline & To many in-app purchases & mani inapp purchas & UserExperience & Neutral \\
\hline & Bad & bad & Rating & Negative \\
\hline & $10+$ & no topic & Other & Neutral \\
\hline \multirow[t]{5}{*}{$\begin{array}{l}\mathrm{D} / \mathrm{L} \\
\text { Free }\end{array}$} & & & & \\
\hline & doesnt work & doesnt work & BugReport & Neutral \\
\hline & Hopefully this works. & work hope & FeatureRequest & Neutral \\
\hline & Still confused & still confus & UserExperience & Negative \\
\hline & cool & cool & Rating & Positive \\
\hline
\end{tabular}


Table A.1 - Continued from previous page

\begin{tabular}{|c|c|c|c|c|}
\hline App Name & Review & Topic & Category & Sentiment \\
\hline & Booty & booti & Other & Neutral \\
\hline \multicolumn{5}{|l|}{ MONOPOLY } \\
\hline \multicolumn{5}{|l|}{ Game } \\
\hline & Thank you for fixing it & fix thank & BugReport & Positive \\
\hline & $\begin{array}{l}\text { Fun game. Could benefit from less pre- } \\
\text { dictable algorithms. }\end{array}$ & $\begin{array}{l}\text { algorithm less predict } \\
\text { game benefit }\end{array}$ & FeatureRequest & Positive \\
\hline & Rigged and confusing & rig confus & UserExperience & Negative \\
\hline & I don't like it & dont like & Rating & Neutral \\
\hline & $-0.99 \$ ? ? ?$ & no topic & Other & Neutral \\
\hline \multicolumn{5}{|l|}{ Timehop } \\
\hline & App won't even open now. & wont even app now open & BugReport & Neutral \\
\hline & $\begin{array}{l}\text { If it added Snapchat story to the list that } \\
\text { would be great }\end{array}$ & $\begin{array}{l}\text { great list stori snapchat } \\
\text { ad }\end{array}$ & FeatureRequest & HighlyPositive \\
\hline & $\begin{array}{l}\text { You should be able to view your Snapchat } \\
\text { memories too }\end{array}$ & $\begin{array}{l}\text { view abl snapchat mem- } \\
\text { ori }\end{array}$ & UserExperience & Neutral \\
\hline & awesome & awesom & Rating & HighlyPositive \\
\hline & Scrolling $¿$ tapping & tap scroll & Other & Neutral \\
\hline \multicolumn{5}{|l|}{ Chatbooks } \\
\hline \multirow{2}{*}{\multicolumn{5}{|c|}{ - Photo }} \\
\hline & & & & \\
\hline & $\begin{array}{l}\text { Keeps going to home page and crashing } \\
\text { please fix!! }\end{array}$ & go keep home pleas fix & BugReport & Positive \\
\hline & $\begin{array}{l}\text { The website could be a little more user } \\
\text { friendly. }\end{array}$ & websit user littl friendli & FeatureRequest & Positive \\
\hline & $\begin{array}{l}\text { I love this app. Love how the books come. } \\
\text { Great quality. }\end{array}$ & $\begin{array}{l}\text { love great app qualiti } \\
\text { book }\end{array}$ & UserExperience & Highly Positive \\
\hline & LOVE IT! & love & Rating & HighlyPositive \\
\hline & Recommended $100 \%$ & recommend & Other & Neutral \\
\hline \multicolumn{5}{|l|}{ Fender Tune } \\
\hline & No problems at all, very intuitive. & problem intuit & BugReport & Negative \\
\hline & $\begin{array}{l}\text { Great basic tuner. Would be great to see the } \\
\text { frequency but still good }\end{array}$ & $\begin{array}{l}\text { great basic tuner see fre- } \\
\text { quenc }\end{array}$ & FeatureRequest & Highly Positive \\
\hline & Simple and easy- super helpful & simpl super help easi & UserExperience & HighlyPositive \\
\hline & Great & great & Rating & HighlyPositive \\
\hline & Gcc & gcc & Other & Neutral \\
\hline \multicolumn{5}{|l|}{ Infinity } \\
\hline \multicolumn{5}{|l|}{ Blade III } \\
\hline & Problems in the game of restar ever & restar problem ever game & BugReport & Negative \\
\hline & It's worth your time please download it & $\begin{array}{l}\text { pleas download worth } \\
\text { time }\end{array}$ & FeatureRequest & Positive \\
\hline & Beautifully hard, genius game & geniu game hard beauti & UserExperience & HighlyPositive \\
\hline & Awesome & awesom & Rating & HighlyPositive \\
\hline & Yessssss & yessssss & Other & Positive \\
\hline \multicolumn{5}{|l|}{$\begin{array}{l}\text { Magic Piano } \\
\text { by Smule }\end{array}$} \\
\hline & $\begin{array}{l}\text { this game is really the best can't stop play- } \\
\text { ing }\end{array}$ & $\begin{array}{l}\text { play stop cant realli } \\
\text { game }\end{array}$ & BugReport & Positive \\
\hline & The adds are annoying! & annoy add & FeatureRequest & HighlyNegative \\
\hline & Too many ads & mani ad & UserExperience & Neutral \\
\hline & Amazing & amaz & Rating & HighlyPositive \\
\hline & :) & no topic & Other & Neutral \\
\hline \multirow{2}{*}{\multicolumn{5}{|c|}{$\begin{array}{l}\text { Chill-Find } \\
\text { Chill Friends }\end{array}$}} \\
\hline & & & & \\
\hline & The stupid app won't let me register. & $\begin{array}{l}\text { let regist mani time ap- } \\
\text { par }\end{array}$ & BugReport & Negative \\
\hline & Could be better & better & FeatureRequest & Neutral \\
\hline & Gotta get em & em gotta get & UserExperience & Neutral \\
\hline & Great & great & Rating & HighlyPositive \\
\hline & & no topic & Other & Neutral \\
\hline
\end{tabular}


Table A.1 - Continued from previous page

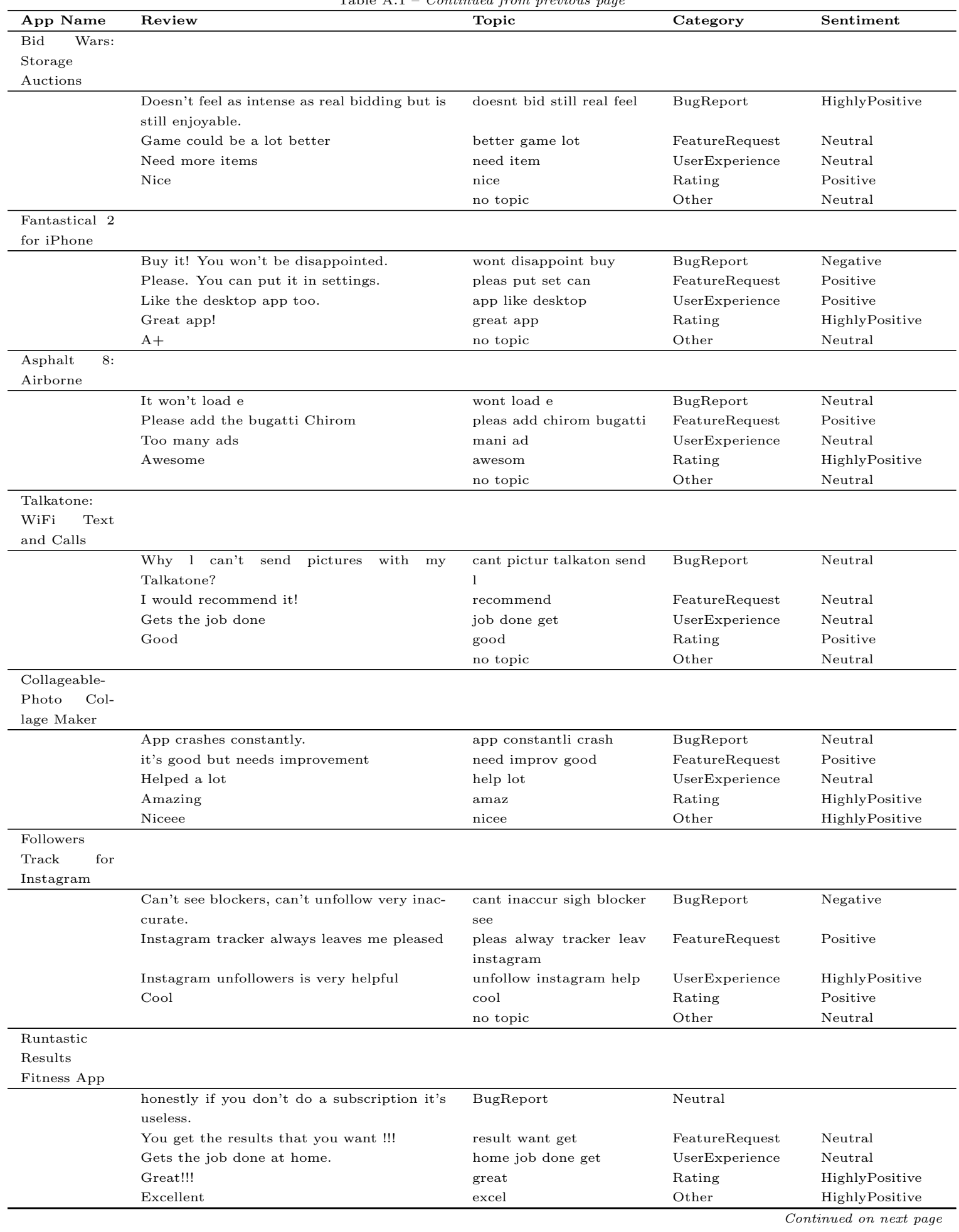


Table A.1 - Continued from previous page

\begin{tabular}{|c|c|c|c|c|}
\hline App Name & Review & Topic & Category & Sentiment \\
\hline \multirow{8}{*}{$\begin{array}{l}\text { AllTrails - } \\
\text { Hiking and } \\
\text { Running }\end{array}$} & & & & \\
\hline & & & & \\
\hline & & & & \\
\hline & $\begin{array}{l}\text { It crashes continuously after updating to the } \\
\text { latest version }\end{array}$ & $\begin{array}{l}\text { updat version crash con- } \\
\text { tinu latest }\end{array}$ & BugReport & Neutral \\
\hline & You guys should add a filter for elevation & filter guy add elev & FeatureRequest & Neutral \\
\hline & all them trails!! and off line too & trail line & UserExperience & Neutral \\
\hline & Love it! & love & Rating & HighlyPositive \\
\hline & & no topic & Other & Neutral \\
\hline \multicolumn{5}{|l|}{4 Pics 1 Word } \\
\hline & The multiplayer mode freezes & multiplay freez mode & BugReport & Neutral \\
\hline & Less ads would be better & better ad less & FeatureRequest & Neutral \\
\hline & Way to many ads. & mani ad way & UserExperience & Neutral \\
\hline & Fun! & fun & Rating & Positive \\
\hline & . & no topic & Other & Neutral \\
\hline \multicolumn{5}{|l|}{ Calculator } \\
\hline & $\begin{array}{l}\text { Terrific calculator- can't think of anything } \\
\text { it can't do }\end{array}$ & $\begin{array}{l}\text { cant calcul think terrif } \\
\text { anyth }\end{array}$ & BugReport & HighlyPositive \\
\hline & $\mathrm{Plz}$ add more languages like "Persian". Tnx & like plz tnx add persian & FeatureRequest & Positive \\
\hline & Very helpful & help & UserExperience & HighlyPositive \\
\hline & Great! & great & Rating & HighlyPositive \\
\hline & Excellent & excel & Other & HighlyPositive \\
\hline \multicolumn{5}{|l|}{ Life Cycle } \\
\hline & $\begin{array}{l}\text { This app solves the mystery of where all } \\
\text { your time is going. }\end{array}$ & solv mysteri app go time & BugReport & Neutral \\
\hline & $\begin{array}{l}\text { Good app and free! Really accurate when it } \\
\text { adds events and locations. }\end{array}$ & add free good accur realli & FeatureRequest & Positive \\
\hline & Super helpful & super help & UserExperience & HighlyPositive \\
\hline & Good & good & Rating & Positive \\
\hline & & no topic & Other & Neutral \\
\hline \multirow{2}{*}{\multicolumn{5}{|c|}{$\begin{array}{l}\text { Napster - } \\
\text { Top Music } \\
\text { and Radio }\end{array}$}} \\
\hline & & & & \\
\hline & Never any problems. Love it & problem never love & BugReport & Negative \\
\hline & $\begin{array}{l}\text { Just give me a die hard, and I would listen } \\
\text { all day! }\end{array}$ & just give die hard day & FeatureRequest & Neutral \\
\hline & Great selection and helpful interface & great interfac help select & UserExperience & HighlyPositive \\
\hline & good & good & Rating & Positive \\
\hline & The & no topic & Other & Neutral \\
\hline \multicolumn{5}{|l|}{ Awesome } \\
\hline \multicolumn{5}{|l|}{ Calendar } \\
\hline \multicolumn{5}{|l|}{ Lite } \\
\hline & Luv you color code it & color code luv & BugReport & HighlyPositive \\
\hline & Add photos to me notes, it is great & note photo add great & FeatureRequest & HighlyPositive \\
\hline & Awesome app. Much help & app much help awesom & UserExperience & HighlyPositive \\
\hline & Great & great & Rating & HighlyPositive \\
\hline & $\mathrm{a}$ & no topic & Other & Neutral \\
\hline \multicolumn{5}{|l|}{ Tayasui } \\
\hline \multicolumn{5}{|l|}{ Sketches Pro } \\
\hline & $\begin{array}{l}\text { Smudging tool doesn't work properly. Few } \\
\text { crashes. }\end{array}$ & $\begin{array}{l}\text { properli crash tool work } \\
\text { doesnt }\end{array}$ & BugReport & Neutral \\
\hline & I just wish there where animation abilities. & wish anim just abil & FeatureRequest & Neutral \\
\hline & if only I knew how to draw... & draw knew & UserExperience & Neutral \\
\hline & GREAT APP & great app & Rating & HighlyPositive \\
\hline & satisfied & satisfi & Other & Positive \\
\hline \multicolumn{5}{|l|}{ BodySpace } \\
\hline \multirow{2}{*}{\multicolumn{5}{|c|}{$\begin{array}{l}-\quad \text { Social } \\
\text { Fitness App }\end{array}$}} \\
\hline & & & & \\
\hline & Solving a common a problem & solv problem common & BugReport & Negative \\
\hline & $\begin{array}{l}\text { Love the community. Need to update / add } \\
\text { workouts. }\end{array}$ & $\begin{array}{l}\text { add communityne love } \\
\text { workout updat }\end{array}$ & FeatureRequest & HighlyPositive \\
\hline
\end{tabular}


Table A.1 - Continued from previous page

\begin{tabular}{|c|c|c|c|c|}
\hline App Name & Review & Topic & Category & Sentiment \\
\hline & Many thanks great apps & mani great thank app & UserExperience & HighlyPositive \\
\hline & Super awesome! & super awesom & Rating & HighlyPositive \\
\hline & :) & no topic & Other & Neutral \\
\hline \multirow{8}{*}{$\begin{array}{l}\text { Smoke Free - } \\
\text { Quit Smok- } \\
\text { ing }\end{array}$} & & & & \\
\hline & & & & \\
\hline & & & & \\
\hline & $\begin{array}{l}\text { This is a great app. And I think it is totally } \\
\text { worth upgrading to the paid version! }\end{array}$ & BugReport & HighlyPositive & \\
\hline & Like this app! Hope there're more GIFs! & gif app like hope therer & FeatureRequest & Positive \\
\hline & It helps! & help & UserExperience & Neutral \\
\hline & awesome! & awesom & Rating & HighlyPositive \\
\hline & & no topic & Other & Neutral \\
\hline \multicolumn{5}{|l|}{$\begin{array}{l}\text { Email - Edi- } \\
\text { son Mail }\end{array}$} \\
\hline & Can't get to my email!!!! & cant email get & BugReport & Neutral \\
\hline & I don't want you to review my emails. & review dont want email & FeatureRequest & Neutral \\
\hline & 2step instructions really confusing & step confus instruct realli & UserExperience & HighlyNegative \\
\hline & Awesome & awesom & Rating & HighlyPositive \\
\hline & Boiii & boiii & Other & Positive \\
\hline \multirow{2}{*}{\multicolumn{5}{|c|}{$\begin{array}{l}\text { InkPad } \\
\text { Notepad - To } \\
\text { do }\end{array}$}} \\
\hline & & & & \\
\hline & $\begin{array}{l}\text { Great app syncs with every device no prob- } \\
\text { lems }\end{array}$ & $\begin{array}{llll}\text { great everi app devic } \\
\text { sync }\end{array}$ & BugReport & HighlyPositive \\
\hline & I would suggest this boss app & suggest app boss & FeatureRequest & Neutral \\
\hline & It's very helpful & help & UserExperience & HighlyPositive \\
\hline & like & like & Rating & Positive \\
\hline & & no topic & Other & Neutral \\
\hline \multirow{2}{*}{\multicolumn{5}{|c|}{$\begin{array}{l}\text { Anytune } \\
\text { Pro+ }\end{array}$}} \\
\hline & & & & \\
\hline & $\begin{array}{l}\text { This app crashes at startup. Please fix. } \\
\text { Thank you. }\end{array}$ & $\begin{array}{l}\text { pleas crash thank app } \\
\text { startup }\end{array}$ & BugReport & Neutral \\
\hline & I wish there was a way to play any song. & wish play way song & FeatureRequest & Neutral \\
\hline & Does everything I needed as a musician. & need everyth musician & UserExperience & Neutral \\
\hline & Amazing & amaz & Rating & HighlyPositive \\
\hline & EXCELLENT!!!!!!! & excel & Other & HighlyPositive \\
\hline \multirow{2}{*}{\multicolumn{5}{|c|}{$\begin{array}{l}\text { Remind: } \\
\text { School Com- } \\
\text { munication }\end{array}$}} \\
\hline & & & & \\
\hline & Can't speak highly enough about this app! & $\begin{array}{l}\text { enough app speak cant } \\
\text { highli }\end{array}$ & BugReport & Neutral \\
\hline & Wish all the classes used this app & wish use class app & FeatureRequest & Neutral \\
\hline & Very helpful and quick & quick help & UserExperience & HighlyPositive \\
\hline & Great & great & Rating & HighlyPositive \\
\hline & & no topic & Other & Neutral \\
\hline \multirow{2}{*}{\multicolumn{5}{|c|}{$\begin{array}{l}\text { Babbel Learn } \\
14 \text { languages }\end{array}$}} \\
\hline & & & & \\
\hline & Mic input doesn't work most of the time. & $\begin{array}{l}\text { doesnt input mic work } \\
\text { time }\end{array}$ & BugReport & Neutral \\
\hline & Please add Japanese & pleas add japanes & FeatureRequest & Positive \\
\hline & Needing to learn Finnish! & need finnish learn & UserExperience & Neutral \\
\hline & Good app! & app good & Rating & Positive \\
\hline & Deleting! & delet & Other & Neutral \\
\hline \multirow{2}{*}{\multicolumn{5}{|c|}{$\begin{array}{l}\text { Algebra } \\
\text { Touch }\end{array}$}} \\
\hline & & & & \\
\hline & $\begin{array}{l}\text { Nothing works, crashes every time. I want } \\
\text { my } \$ 2.00 \text { back, I DEMAND IT!!! }\end{array}$ & $\begin{array}{l}\text { time back crash demand } \\
\text { want }\end{array}$ & BugReport & Neutral \\
\hline & Just wish there were more levels. & wish just level & FeatureRequest & Neutral \\
\hline & Great help & great help & UserExperience & HighlyPositive \\
\hline & Great & great & Rating & HighlyPositive \\
\hline
\end{tabular}


Table A.1 - Continued from previous page

\begin{tabular}{|c|c|c|c|c|}
\hline App Name & Review & Topic & Category & Sentiment \\
\hline & More! & no topic & Other & Neutral \\
\hline \multicolumn{5}{|l|}{ StarMap } \\
\hline \multicolumn{5}{|l|}{$3 \mathrm{D}+$} \\
\hline & I can't say enough about this app! & app cant say enough & BugReport & Neutral \\
\hline & $\begin{array}{l}\text { Amazing app, one suggestion though. You } \\
\text { guys should add exoplanets! }\end{array}$ & $\begin{array}{l}\text { guy one exoplanet } \\
\text { though add }\end{array}$ & FeatureRequest & HighlyPositive \\
\hline & Compass is reversed. Help! & revers help compass & UserExperience & Neutral \\
\hline & COOL! & cool & Rating & Positive \\
\hline & & no topic & Other & Neutral \\
\hline
\end{tabular}


Table A.2: Selected 35 apps details.

\begin{tabular}{|c|c|c|c|}
\hline Category & Name & Price & Release Date \\
\hline \multicolumn{4}{|l|}{ Games } \\
\hline & MONOPOLY Game & 0.99 & 20 Nov,2009 \\
\hline & Infinity Blade III & 6.99 & 18 Sep,2013 \\
\hline & Bid Wars: Storage Auctions & 0 & 05 Nov, 2015 \\
\hline & Asphalt 8: Airborne & 0 & 22 Aug,2013 \\
\hline & 4 Pics 1 Word & 0 & 04 Feb,2013 \\
\hline \multicolumn{4}{|c|}{ Social Networking } \\
\hline & Followers - Social Analytics For Instagram & 0 & $04 \mathrm{Sep}, 2012$ \\
\hline & Timehop & 0 & 22 Oct, 2012 \\
\hline & Chill-Find Chill Friends with Same InterestsandViews & 0 & 11 Jun, 2017 \\
\hline & Talkatone: WiFi Text and Calls & 0 & 27 Oct, 2010 \\
\hline & Followers Track for Instagram & 0 & 28 Jun,2017 \\
\hline \multicolumn{4}{|c|}{ Health and Fitness } \\
\hline & Runtastic Results Fitness App & 0 & 12 Nov,2015 \\
\hline & AllTrails - Hiking and Running & 0 & 17 Dec, 2010 \\
\hline & Life Cycle - Track Your Time Automatically & 0 & 20 Jan, 2016 \\
\hline & BodySpace - Social Fitness App & 0 & 07 Sep,2013 \\
\hline & Smoke Free - Quit Smoking Now & 0 & 26 Feb,2013 \\
\hline \multicolumn{4}{|l|}{ Productivity } \\
\hline & Fantastical 2 for iPhone & 4.99 & 30 Oct,2013 \\
\hline & Awesome Calendar Lite & 0 & 06 Dec,2011 \\
\hline & Tayasui Sketches Pro & 4.99 & $31 \mathrm{Dec}, 2014$ \\
\hline & Email - Edison Mail & 0 & 27 Apr, 2016 \\
\hline & InkPad Notepad - Notes - To do & 0 & $27 \mathrm{Jul}, 2015$ \\
\hline \multicolumn{4}{|c|}{ Photo and Video } \\
\hline & Instasave - Grab from Instagram Photos and Repost It & 0 & $27 \mathrm{Sep}, 2015$ \\
\hline & Add Background Music To Video & 0 & $16 \mathrm{Jul}, 2015$ \\
\hline & D/L Plus Free - Get and Save Files From Web Browser and File Manager & 0 & 08 Jan,2016 \\
\hline & Chatbooks - Photo Books & 0 & 06 Nov, 2013 \\
\hline & Collageable - Photo Collage Maker, Pic Grid Free & 0 & $12 \mathrm{Apr}, 2016$ \\
\hline \multicolumn{4}{|l|}{ Education } \\
\hline & Calculator & 2.99 & 15 Sep,2011 \\
\hline & Remind: School Communication & 0 & 02 May,2012 \\
\hline & Babbel Learn 14 languages & 0 & 11 Jun,2014 \\
\hline & Algebra Touch & 2.99 & 02 Aug,2010 \\
\hline & StarMap 3D+: Night Sky, Astronomy, Star View Guide & 2.99 & 25 Jan, 2010 \\
\hline \multicolumn{4}{|l|}{ Music } \\
\hline & Saavn Music and Radio & 0 & 08 Jun,2011 \\
\hline & Fender Tune & 0 & 05 Aug,2016 \\
\hline & Magic Piano by Smule & 0 & 04 May,2011 \\
\hline & Napster - Top Music and Radio & 0 & 14 Dec,2012 \\
\hline & Anytune Pro+ & 14.99 & 09 Dec,2011 \\
\hline
\end{tabular}




\section{Appendix B}

\section{Figures}

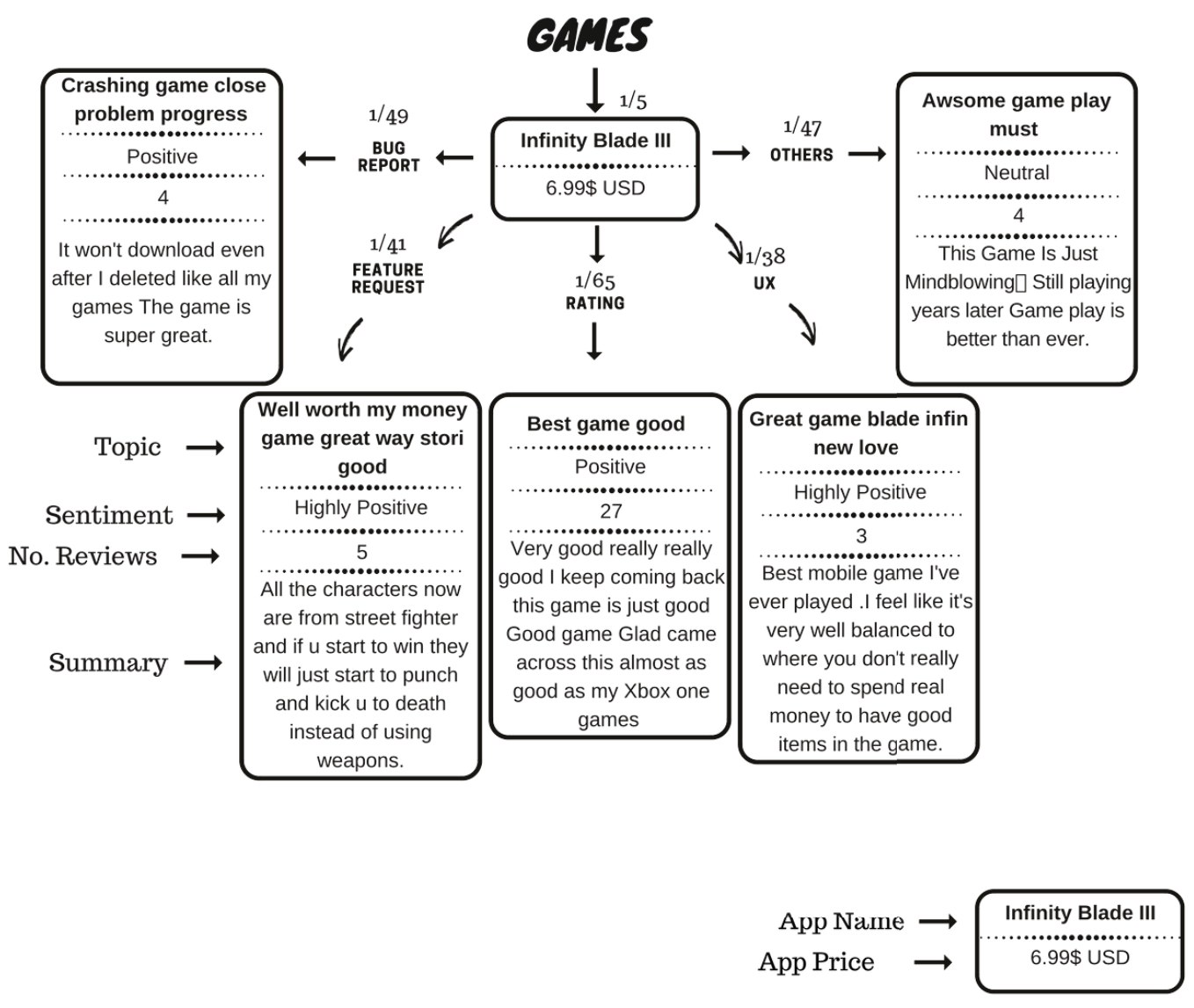

Figure B.1: Cluster results, Page 1 


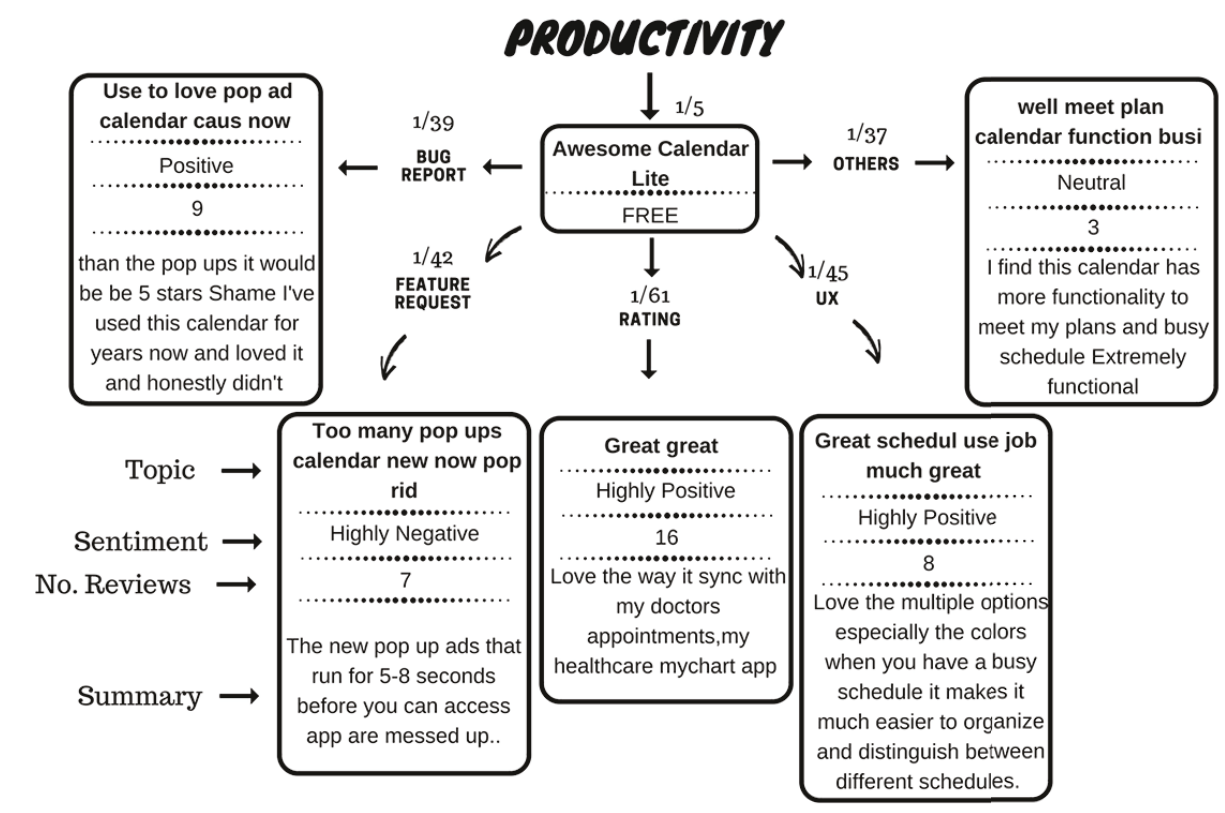

\section{SOCIAL NETWORKING}

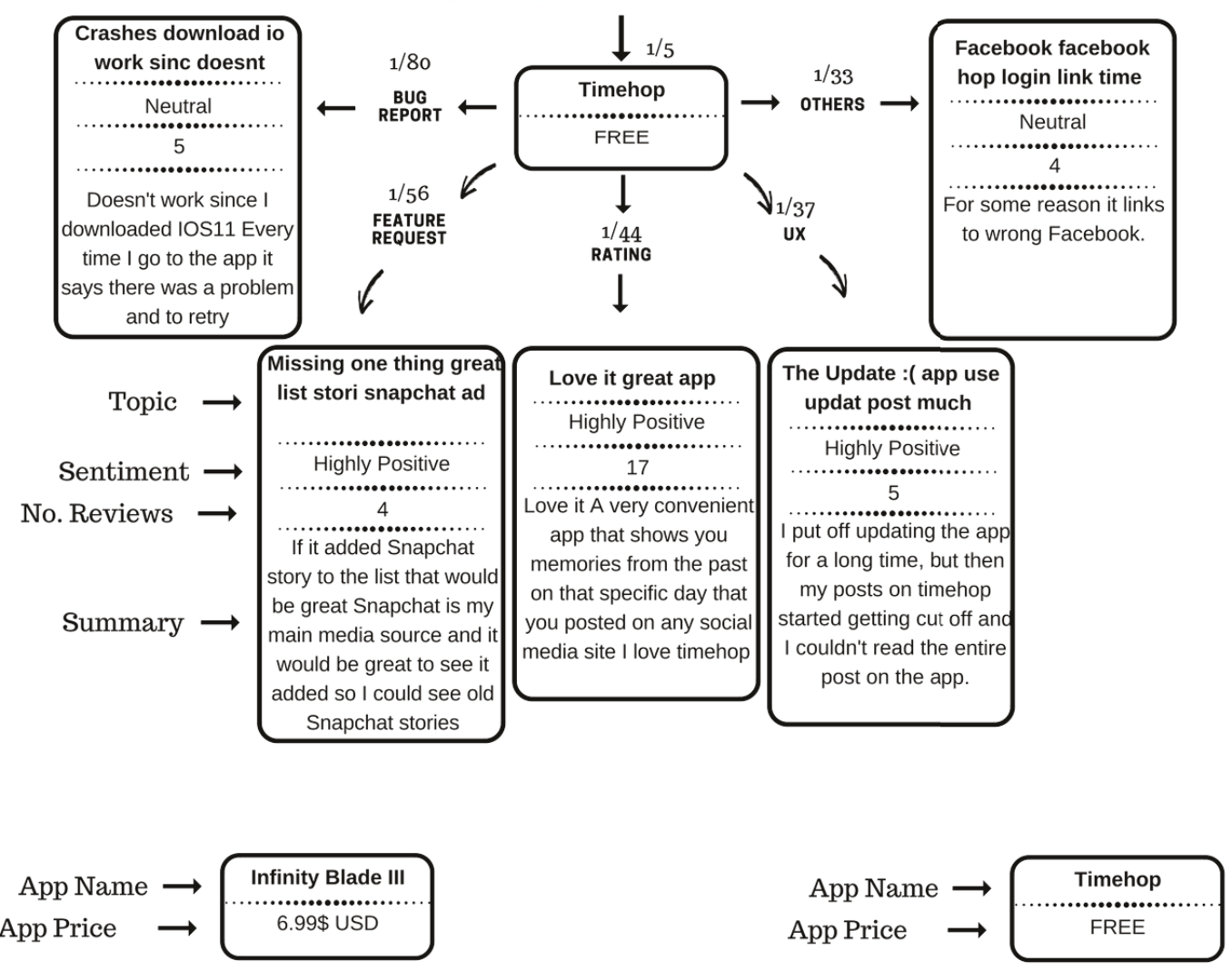

Figure B.2: Cluster results, Page 2 

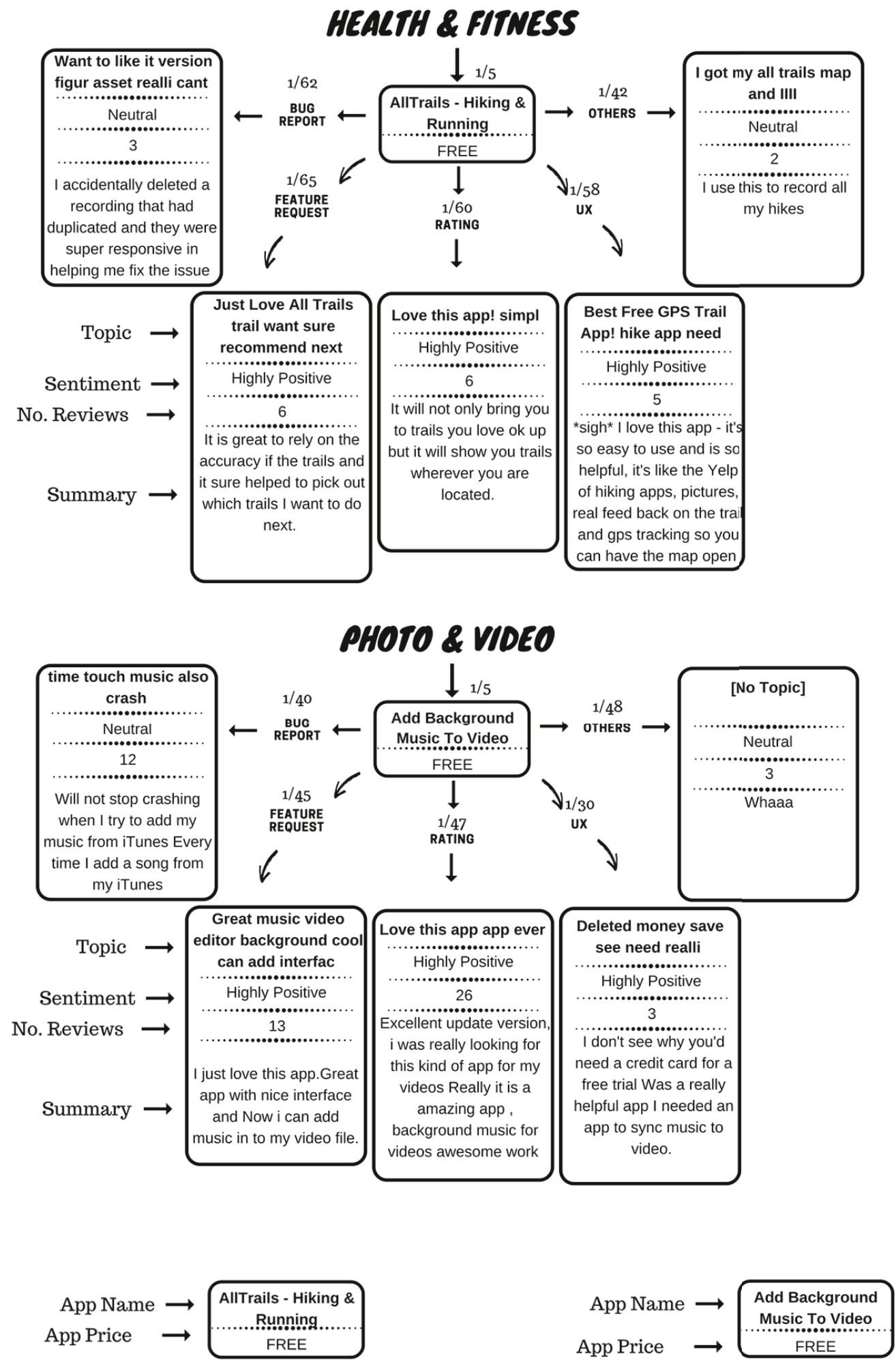

Figure B.3: Cluster results, Page 3 

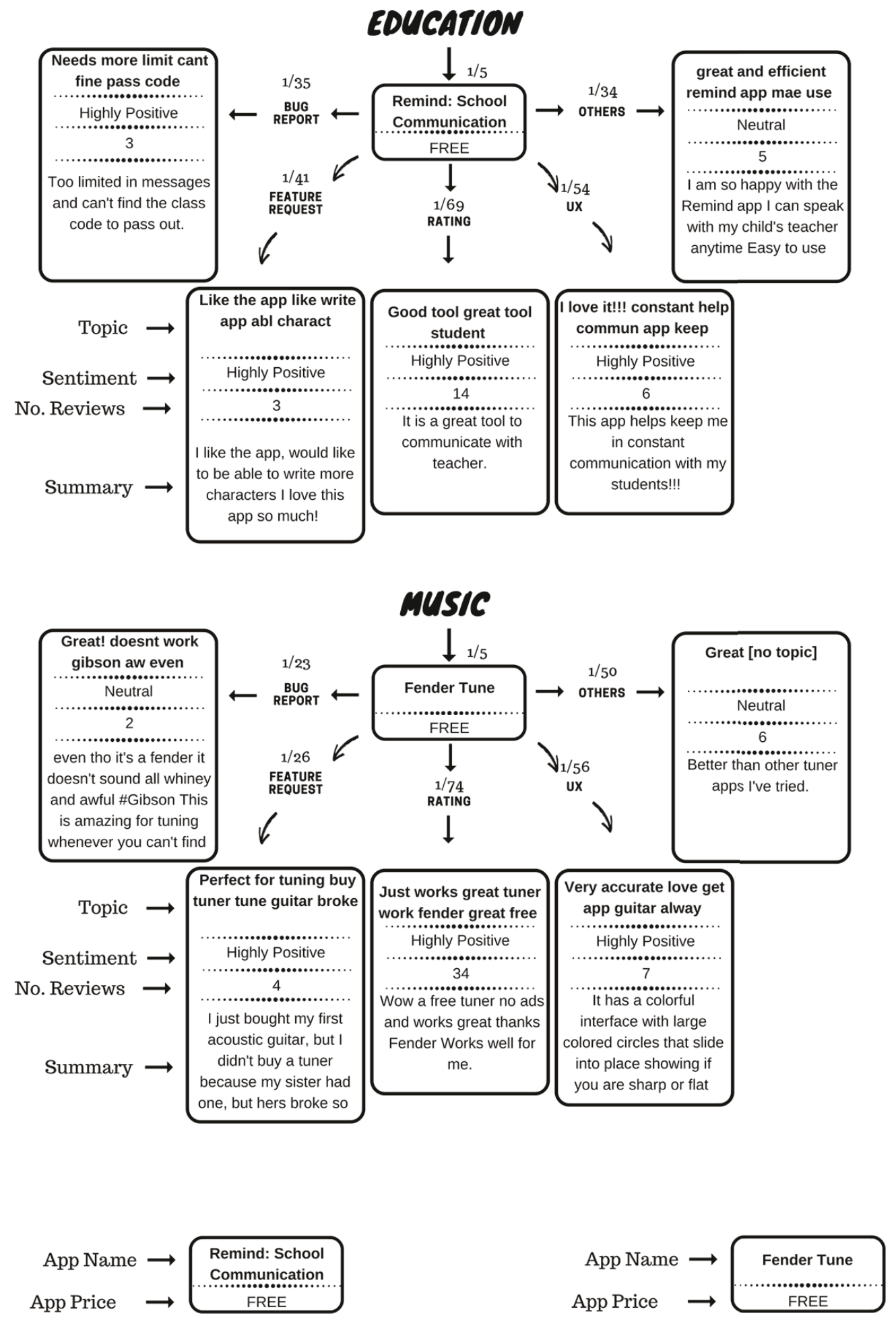

Figure B.4: Cluster results, Page 4 


\section{Bibliography}

[1] Dennis Pagano and Walid Maalej. User feedback in the appstore: An empirical study. In Requirements Engineering Conference (RE), 2013 21st IEEE International, pages 125-134. IEEE, 2013.

[2] David M Blei, Andrew Y Ng, and Michael I Jordan. Latent dirichlet allocation. Journal of machine Learning research, 3(Jan):993-1022, 2003.

[3] Ricardo Baeza-Yates, Berthier Ribeiro-Neto, et al. Modern information retrieval, volume 463. ACM press New York, 1999.

[4] Peter D Turney and Patrick Pantel. From frequency to meaning: Vector space models of semantics. Journal of artificial intelligence research, 37:141-188, 2010.

[5] N Rotem. Open text summarizer (ots). Retrieved July, 3(2006):2006, 2003.

[6] Apple Inc. App store. https://www. apple.com/appstore, 2017.

[7] Google. Play store. https://play.google.com/store?hl=en, 2017.

[8] Amazon.com. Amazon appstore. https://www.amazon.com/mobile-apps/, 2017.

[9] Sebastiano Panichella, Andrea Di Sorbo, Emitza Guzman, Corrado A Visaggio, Gerardo Canfora, and Harald C Gall. How can i improve my app? classifying user reviews for software maintenance and evolution. In Software maintenance and evolution (ICSME), 2015 IEEE international conference on, pages 281-290. IEEE, 2015. 
[10] Emitza Guzman and Walid Maalej. How do users like this feature? a fine grained sentiment analysis of app reviews. In Requirements Engineering Conference (RE), 2014 IEEE 22nd International, pages 153-162. IEEE, 2014.

[11] Walid Maalej and Dennis Pagano. On the socialness of software. In Dependable, Autonomic and Secure Computing (DASC), 2011 IEEE Ninth International Conference on, pages 864-871. IEEE, 2011.

[12] Norbert Seyff, Florian Graf, and Neil Maiden. Using mobile re tools to give end-users their own voice. In Requirements Engineering Conference (RE), 2010 18th IEEE International, pages 37-46. IEEE, 2010.

[13] Rishi Chandy and Haijie Gu. Identifying spam in the ios app store. In Proceedings of the 2Nd Joint WICOW/AIRWeb Workshop on Web Quality, WebQuality '12, pages 56-59, New York, NY, USA, 2012. ACM.

[14] Pär Carlshamre, Kristian Sandahl, Mikael Lindvall, Björn Regnell, and J Natt och Dag. An industrial survey of requirements interdependencies in software product release planning. In Requirements Engineering, 2001. Proceedings. Fifth IEEE International Symposium on, pages 84-91. IEEE, 2001.

[15] Pär Carlshamre. Release planning in market-driven software product development: Provoking an understanding. Requirements engineering, 7(3):139-151, 2002.

[16] Antônio Mauricio Pitangueira, Rita Suzana P Maciel, and Márcio Barros. Software requirements selection and prioritization using sbse approaches: A systematic review and mapping of the literature. Journal of Systems and Software, 103:267-280, 2015.

[17] Davoud Mougouei and David MW Powers. Dependency-aware software release planning through mining user preferences. arXiv preprint arXiv:1702.05592, 2017. 
[18] Lorenzo Villarroel, Gabriele Bavota, Barbara Russo, Rocco Oliveto, and Massimiliano Di Penta. Release planning of mobile apps based on user reviews. In Proceedings of the 38th International Conference on Software Engineering, pages 14-24. ACM, 2016.

[19] Eya Ben Charrada. Which one to read? factors influencing the usefulness of online reviews for re. In Requirements Engineering Conference Workshops (REW), IEEE International, pages 46-52. IEEE, 2016.

[20] Ning Chen, Jialiu Lin, Steven CH Hoi, Xiaokui Xiao, and Boshen Zhang. Arminer: mining informative reviews for developers from mobile app marketplace. In Proceedings of the 36th International Conference on Software Engineering, pages 767-778. ACM, 2014.

[21] Emitza Guzman, Muhammad El-Haliby, and Bernd Bruegge. Ensemble methods for app review classification: An approach for software evolution (n). In Automated Software Engineering (ASE), 2015 30th IEEE/ACM International Conference on, pages 771-776. IEEE, 2015.

[22] Jeungmin Oh, Daehoon Kim, Uichin Lee, Jae-Gil Lee, and Junehwa Song. Facilitating developer-user interactions with mobile app review digests. In CHI'13 Extended Abstracts on Human Factors in Computing Systems, pages 1809-1814. ACM, 2013.

[23] Adelina Ciurumelea, Andreas Schaufelbühl, Sebastiano Panichella, and Harald C Gall. Analyzing reviews and code of mobile apps for better release planning. In Software Analysis, Evolution and Reengineering (SANER), 2017 IEEE 24th International Conference on, pages 91-102. IEEE, 2017.

[24] Timo Johann, Christoph Stanik, Walid Maalej, et al. Safe: A simple approach for feature extraction from app descriptions and app reviews. In Requirements Engineering Conference (RE), 2017 IEEE 25th International, pages 21-30. IEEE, 2017. 
[25] Maleknaz Nayebi, Henry Cho, Homayoon Farrahi, and Guenther Ruhe. App store mining is not enough. In Software Engineering Companion (ICSE-C), 2017 IEEE/ACM 39th International Conference on, pages 152-154. IEEE, 2017.

[26] Iván Tactuk Mercado, Nuthan Munaiah, and Andrew Meneely. The impact of cross-platform development approaches for mobile applications from the user's perspective. In Proceedings of the International Workshop on App Market Analytics, pages 43-49. ACM, 2016.

[27] Walid Maalej and Hadeer Nabil. Bug report, feature request, or simply praise? on automatically classifying app reviews. In Requirements Engineering Conference (RE), 2015 IEEE 23rd International, pages 116-125. IEEE, 2015.

[28] Claudia Iacob and Rachel Harrison. Retrieving and analyzing mobile apps feature requests from online reviews. In Mining Software Repositories (MSR), 2013 10th IEEE Working Conference on, pages 41-44. IEEE, 2013.

[29] Claudia Iacob, Shamal Faily, and Rachel Harrison. Maram: Tool support for mobile app review management. In Proceedings of the 8th EAI International Conference on Mobile Computing, Applications and Services, pages 42-50. ICST (Institute for Computer Sciences, Social-Informatics and Telecommunications Engineering), 2016.

[30] Github. Github. https://github.com/, 2017.

[31] ATLASSIAN. Jira. https://www.atlassian.com/software/jira, 2017.

[32] BugZilla. Bugzilla. https://www. bugzilla.org, 2017.

[33] Bing Liu. Sentiment analysis and opinion mining. Synthesis lectures on human language technologies, 5(1):1-167, 2012.

[34] Alexander Pak and Patrick Paroubek. Twitter as a corpus for sentiment analysis and opinion mining. In $L R E c$, volume 10, 2010. 
[35] Apoorv Agarwal, Boyi Xie, Ilia Vovsha, Owen Rambow, and Rebecca Passonneau. Sentiment analysis of twitter data. In Proceedings of the workshop on languages in social media, pages 30-38. Association for Computational Linguistics, 2011.

[36] Anastasia Giachanou and Fabio Crestani. Tracking sentiment by time series analysis. In Proceedings of the 39th International ACM SIGIR conference on Research and Development in Information Retrieval, pages 1037-1040. ACM, 2016.

[37] Anastasia Giachanou, Ida Mele, and Fabio Crestani. Explaining sentiment spikes in twitter. In Proceedings of the 25th ACM International on Conference on Information and Knowledge Management, pages 2263-2268. ACM, 2016.

[38] Robbert Jongeling, Proshanta Sarkar, Subhajit Datta, and Alexander Serebrenik. On negative results when using sentiment analysis tools for software engineering research. Empirical Software Engineering, pages 1-42.

[39] Mike Thelwall, Kevan Buckley, Georgios Paltoglou, Di Cai, and Arvid Kappas. Sentiment strength detection in short informal text. Journal of the American Society for Information Science and Technology, 61(12):2544-2558, 2010.

[40] Bin Fu, Jialiu Lin, Lei Li, Christos Faloutsos, Jason Hong, and Norman Sadeh. Why people hate your app: Making sense of user feedback in a mobile app store. In Proceedings of the 19th ACM SIGKDD international conference on Knowledge discovery and data mining, pages 1276-1284. ACM, 2013.

[41] Claudia Iacob and Rachel Harrison. Retrieving and analyzing mobile apps feature requests from online reviews. In Mining Software Repositories (MSR), 2013 10th IEEE Working Conference on, pages 41-44. IEEE, 2013.

[42] Alessandra Gorla, Ilaria Tavecchia, Florian Gross, and Andreas Zeller. Checking app behavior against app descriptions. In Proceedings of the 36th International Conference on Software Engineering, pages 1025-1035. ACM, 2014. 
[43] Gediminas Adomavicius and Alexander Tuzhilin. Toward the next generation of recommender systems: A survey of the state-of-the-art and possible extensions. IEEE Transactions on Knowledge and Data Engineering, 17(6):734-749, 2005.

[44] Anand Rajaraman and Jeffrey David Ullman. Mining of Massive Datasets. Cambridge University Press, 2011.

[45] Joeran Beel, Bela Gipp, Stefan Langer, and Corinna Breitinger. Research-paper recommender systems: a literature survey. International Journal on Digital Libraries, 17(4):305-338, Nov 2016.

[46] M.F. Porter. An algorithm for suffix stripping. Program, 14(3):130-137, 1980.

[47] Graham A Stephen. String searching algorithms, volume 3. World Scientific, 1994. 